\title{
Energy Integration Describes Sound-Intensity Coding in an Insect Auditory System
}

\author{
Tim Gollisch, Hartmut Schütze, Jan Benda, and Andreas V. M. Herz \\ Institute for Theoretical Biology, Department of Biology, Humboldt University, 10115 Berlin, Germany
}

We investigate the transduction of sound stimuli into neural responses and focus on locust auditory receptor cells. As in other mechanosensory model systems, these neurons integrate acoustic inputs over a fairly broad frequency range. To test three alternative hypotheses about the nature of this spectral integration (amplitude, energy, pressure), we perform intracellular recordings while stimulating with superpositions of pure tones. On the basis of online data analysis and automatic feedback to the stimulus generator, we systematically explore regions in stimulus space that lead to the same level of neural activity. Focusing on such iso-firing-rate regions allows for a rigorous quantitative comparison of the electrophysiological data with predictions from the three hypotheses that is independent of nonlinearities induced by the spike dynamics. We find that the dependence of the firing rates of the receptors on the composition of the frequency spectrum can be well described by an energy-integrator model. This result holds at stimulus onset as well as for the steady-state response, including the case in which adaptation effects depend on the stimulus spectrum. Predictions of the model for the responses to bandpass-filtered noise stimuli are verified accurately. Together, our data suggest that the sound-intensity coding of the receptors can be understood as a three-step process, composed of a linear filter, a summation of the energy contributions in the frequency domain, and a firing-rate encoding of the resulting effective sound intensity. These findings set quantitative constraints for future biophysical models.

Key words: mechanosensory transduction; spectral integration; auditory receptor; hearing; sound intensity; energy; model; locust
Auditory receptor cells are commonly characterized by their responses to pure tones. For example, threshold curves characterize the minimum intensity needed to evoke a response as a function of the frequency of a pure tone; rate-intensity functions describe how the response depends on the tone's intensity. Natural signals, however, are only rarely restricted to single frequencies, and receptor cells often show a broad frequency tuning. Our understanding of auditory coding is thus not satisfactory as long as we do not know how the relative intensities of different frequencies contained in a sound signal are integrated by auditory receptors. Investigating this spectral integration helps us also to scrutinize basic principles of the mechanosensory transduction process.

In general, the response of the receptor could be any complicated, nonlinear function of the frequency spectrum. One may hope, however, that the underlying mechanism is simple enough to allow for a straightforward phenomenological description. One such way of combining different spectral contents would be the extraction of a single physical stimulus property. Its nature is intensely debated with respect to the question of temporal integration, i.e., how stimulus intensities are combined over time. Psychoacoustic measurements of intensity-duration tradeoffs sug-

\footnotetext{
Received May 1, 2002; revised Aug. 13, 2002; accepted Sept. 9, 2002.

This work was supported by Boehringer Ingelheim Fonds (T.G.) and the Deutsche Forschungsgemeinschaft. We are grateful to Christian Machens and Martin Stemmler for fruitful discussions and Peter Heil, Matthias Hennig, and Rüdiger von der Heydt for valuable comments on this manuscript.

Correspondence should be addressed to Andreas V. M. Herz, Institute for Theoretical Biology, Department of Biology, Humboldt University, 10115 Berlin, Germany. E-mail: herz@itb.biologie.hu-berlin.de.

H. Schütze's present address: Krieger Mind/Brain Institute, Johns Hopkins University, Baltimore, MD 21218.

J. Benda's present address: Department of Physics, University of Ottawa, Ottawa, Ontario, Canada K1N 6N5.

Copyright (C) 2002 Society for Neuroscience $\quad 0270-6474 / 02 / 2210434-15 \$ 15.00 / 0$
}

gest that the stimulus energy is the crucial variable (Garner, 1947; Plomp and Bouman, 1959; Zwislocki, 1965; Florentine et al., 1988), while a recent investigation of first-spike latencies in mammalian auditory-nerve fibers finds the time-integrated pressure as the decisive stimulus attribute (Heil and Neubauer, 2001). In insect auditory receptors, the differences between thresholds for one- and two-click stimuli and intensity-duration tradeoffs are consistent with temporal energy integration (Tougaard, 1996, 1998). Care must be taken, however, in the interpretation of these data because temporal integration also depends on the time course of several biophysical processes after the primary signal transduction such as internal calcium dynamics and spike generation.

Spectral integration, on the other hand, depends at least in insects almost exclusively on the mechanosensory transduction process; any fluctuations on the several kilohertz scale of relevant sound frequencies that were still present after the transduction (i.e., in the cell-membrane conductance) would be highly attenuated by the low-pass filter properties of the cell membrane (Koch, 1999). Looking at spectral integration instead of temporal integration therefore enables us to focus on the site of primary signal transduction.

For these reasons, we develop a descriptive model for the responses of auditory receptor neurons to stationary stimuli with arbitrary power spectrum. The model comprises three steps, which correspond to the coupling, the transduction, and the encoding of the primary signal (Eyzaguirre and Kuffler, 1955; French, 1992). Focusing on the locust auditory system, we investigate three alternative hypotheses about which stimulus property governs the transduction process: the maximum amplitude of the stimulus, the stimulus energy, and the average half-wave-rectified signal amplitude. To test the model framework and distinguish between the rival hypotheses, intracellular recordings from the axons of recep- 
tor cells are performed. Based on a systematic exploration of stimuli that cause identical neural responses, the recordings reveal how the individual spectral contributions are integrated into one effective sound intensity.

\section{MATERIALS AND METHODS}

Electrophysiology. All experiments were performed on adult Locusta migratoria. The tympanal auditory organ of these animals is located in the first abdominal segment. After decapitation, removal of the legs, wings, intestines, and the dorsal part of the thorax, the animal was waxed to a holder, and the metathoracic ganglion and auditory nerve were exposed. Action potentials from auditory receptor cells were recorded intracellularly in the auditory nerve with standard glass microelectrodes (borosilicate, GC100F-10; Harvard Apparatus Ltd., Edenbridge, UK) filled with a $1 \mathrm{M} \mathrm{KCl}$ solution (50-110 $\mathrm{M} \Omega$ resistance). The signals were amplified (BRAMP-01; NPI Electronic, Tamm, Germany) and recorded by a data acquisition board (PCI-MIO-16E-1; National Instruments, München, Germany) with a sampling rate of $10 \mathrm{kHz}$. Detection of action potentials and generation of acoustic signals were controlled on-line by the custom-made Online Electrophysiology Laboratory (OEL) software. Stimuli were transmitted by the above-mentioned data acquisition board with a conversion rate of $100 \mathrm{kHz}$ to the loudspeakers [Esotec D-260, Dynaudio (Skanderborg, Denmark) on a DCA 450 amplifier (Denon Electronic $\mathrm{GmbH}$, Ratingen, Germany)]. These were mounted at $30 \mathrm{~cm}$ distance on each side of the animal so that the incidence of soundpressure waves was orthogonal to the body axis. Stimuli were played only by the loudspeaker ipsilateral to the recorded auditory nerve. The linearity of the loudspeakers for superpositions of multiple tones was verified by playing samples of the stimuli used in the experiments while recording the sound at the site of the animals with a high-precision microphone [40AC, G.R.A.S. Sound \& Vibration (Vedbæk, Denmark) on a 2690 conditioning amplifier (Brüel \& Kjær, Langen, Germany)]. During the experiments, animals were kept either at room temperature, which was $\sim 20^{\circ} \mathrm{C}$ or at a constant temperature of $30^{\circ} \mathrm{C}$. No systematic trends regarding a possible temperature dependence of the studied phenomena were observed. All experiments were performed in a Faraday cage lined with sound-attenuating foam to reduce echoes. Recordings from 45 receptor cells stemming from 18 animals (with at most 4 cells from the same animal) were used in this study.

The experimental protocol complied with German law governing animal care.

Measurement of rate-intensity functions. In general, each sound stimulus was presented for a duration of $100 \mathrm{msec}$, separated by pauses of at least $400 \mathrm{msec}$. To investigate adaptation effects, control experiments with longer stimuli and pauses $(300 / 500 \mathrm{msec}$ or $500 / 750 \mathrm{msec})$ were performed. All of these stimuli are decidedly longer than typical integration times of insect auditory receptors (1-3 msec as determined by reverse correlation for locust auditory receptors; data not shown) (see also Tougaard, 1998). Responses were measured by the average firing rate, calculated as the total number of spikes divided by the stimulus length. Spikes were detected on-line and counted from stimulus onset until $20 \mathrm{msec}$ beyond stimulus offset to include all spikes elicited by the stimulus. This is justified because the investigated cells show no or only very low spontaneous activity and no offset response. Spike trains from the control experiments were also used for off-line analysis of specific response episodes.

Rate-intensity functions were determined in the following way. First, the stimulus was presented in steps of $5 \mathrm{~dB}$ between 20 and $100 \mathrm{~dB}$ sound pressure level (SPL) (for a definition see Eq. 21 in the Appendix) to obtain the general shape of the rate-intensity function. These data were used to identify the intensity range that gave rise to firing rates between 50 and $250 \mathrm{~Hz}$. Within this dynamic range of $\sim 10-15 \mathrm{~dB}$, additional measurements in steps of 1 or $2 \mathrm{~dB}$ were performed, and these were repeated 4-10 times to yield average firing rates and their SDs.

Stimulus intensities corresponding to given firing rates were obtained by fitting a straight line through the four points closest to the desired firing rate as shown in Figure 1. Errors on these measurements follow from the errors of the fitted parameters according to the law of error propagation. Thresholds were determined by linear extrapolation to zero firing rate from data points with a low, but significant firing rate.

Superposition of pure tones. Measuring rate-intensity functions for pure tones allows one to understand how the firing rate $r$ depends on the amplitude $A$ of a single tone for a certain sound frequency, $r=r(A)$. Investigating spectral integration amounts to asking whether this under-
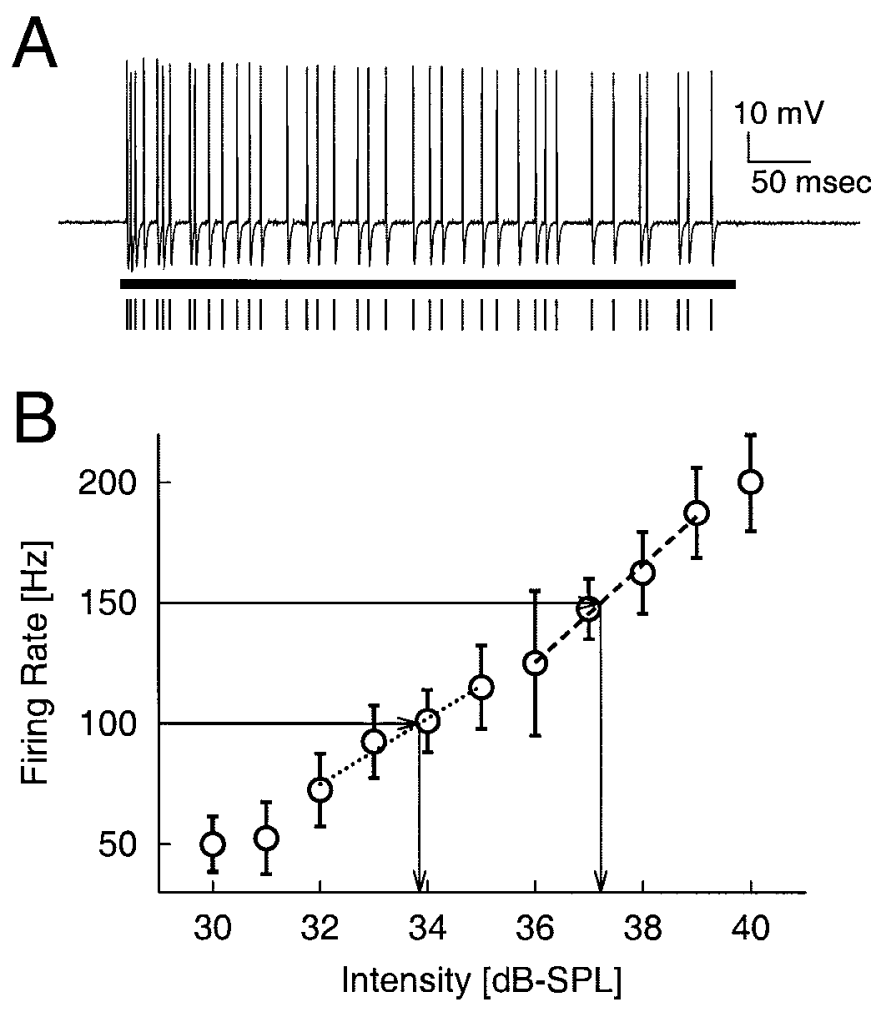

Figure 1. Determination of sound intensities corresponding to given firing rates. $A$, Example of a spike train recorded intracellularly from an axon of a receptor cell. Calibration is given to the right. The thick bar below the voltage trace denotes the $500 \mathrm{msec}$ pure-tone stimulus. The vertical bars below show the spike times as determined by the spikedetection algorithm. The firing rate is calculated by counting the spikes and averaging over several stimulus repetitions. $B$, Example of the rising part of a rate-intensity function $(\bigcirc)$ measured in steps of $1 \mathrm{~dB}$. Each stimulus was repeated multiple times. Vertical bars denote the SD of each measurement. Linear fits through the four points closest to the firing rates of interest, here 100 and $150 \mathrm{~Hz}$, are depicted as dotted and dashed lines, respectively. The arrows indicate the readout of the corresponding intensities.

standing can be extended to stimuli that contain multiple tones simultaneously. We therefore try to obtain a description of the firing rate $r$ depending on the amplitudes $A_{1}, A_{2}, \ldots$ of the different frequency components of such stimuli, $r=r\left(A_{1}, A_{2}, \ldots\right)$.

In a first set of experiments, stimuli were sound-pressure waves $S(t)$ consisting of two or three pure tones of amplitudes $A_{\mathrm{n}}$, frequencies $f_{\mathrm{n}}$, and phase offsets $\varphi_{\mathrm{n}}, n=1,2,3$ :

$$
S(t)=A_{1} \sin \left(2 \pi f_{1} t+\varphi_{1}\right)+A_{2} \sin \left(2 \pi f_{2} t+\varphi_{2}\right)+A_{3} \sin \left(2 \pi f_{3} t+\varphi_{3}\right),
$$

with $A_{3}=0$ for the two-tone experiments. We used stimuli that were far longer than the periods of the sine waves and avoided combinations of frequencies that are related to each other by small integer factors. This makes the measurements insensitive to the relative phases of the individual sine tones, which we cannot control in our experiments because of putative phase shifts at the tympanal membrane (Michelsen, 1971b). For concreteness, we set $\varphi_{1}=\varphi_{2}=\varphi_{3}=0$ in all experiments. The frequencies were chosen to be far enough apart to avoid beating. The two-tone experiments were performed with sound frequencies $f_{1}=4 \mathrm{kHz}$ and $f_{2}=$ $3 / \pi \cdot 10 \mathrm{kHz} \approx 9.55 \mathrm{kHz}$, the three-tone experiments with $f_{1}=4 \mathrm{kHz}$, $f_{2}=3 / \pi \cdot 10 \mathrm{kHz} \approx 9.55 \mathrm{kHz}$, and $f_{3}=10 / \pi^{2} \cdot 15 \mathrm{kHz} \approx 15.20 \mathrm{kHz}$ or with $f_{1}=6 \mathrm{kHz}, f_{2}=3 / \pi \cdot 9 \mathrm{kHz} \approx 8.59 \mathrm{kHz}$, and $f_{3}=10 / \pi^{2} \cdot 17 \mathrm{kHz} \approx 17.22$ $\mathrm{kHz}$.

Within the present approach, we are concerned only with the encoding of sound intensity and not with temporal aspects. We thus restricted our attention to stationary stimuli with constant envelope as described above. 
This is justified because the responses of locust auditory receptors do not phase lock to sound frequencies in the kilohertz range (Suga, 1960; Hill, 1983a).

The experiments were designed to identify, for individual receptors, sets of amplitude combinations $\left(A_{1}, A_{2}\right)$ or $\left(A_{1}, A_{2}, A_{3}\right)$, respectively, that result in the same firing rate. The recorded data were analyzed within a model framework, which includes explicit predictions about how these amplitude combinations should be related to each other. In Results, the model is systematically developed and discussed. Here, we only present the main aspects and cover technical issues and questions regarding the model's role within the data analysis.

In summary, we compute the average firing rate of a receptor cell in the following three-step process.

(1) The stimulus is a sound-pressure wave $S(t)$, a superposition of pure tones with frequencies $f_{n}$, amplitudes $A_{n}$, and phase offsets $\varphi_{n}, S(t)=$ $\sum_{n=1}^{N} A_{n} \sin \left(2 \pi f_{n} t+\varphi_{n}\right)$. In the first step, this signal is linearly filtered and thereby turned into:

$$
\tilde{S}(t)=\sum_{n=1}^{N} \frac{A_{n}}{C_{n}} \sin \left(2 \pi f_{n} t+\tilde{\varphi}_{n}\right)
$$

This means that every tone receives a gain factor $1 / C_{n}$. In addition, the phase may change from $\varphi_{n}$ to $\tilde{\varphi}_{n}$. The inverse of the filter constant $C_{n}$ thus corresponds to the sensitivity for the frequency $f_{n}$ : the smaller the $C_{n}$, the more sensitive the receptor at the corresponding sound frequency.

(2) An effective sound intensity $J$ is computed according to one of the following three hypotheses:

$$
\begin{aligned}
& \text { amplitude hypothesis }(\mathrm{AH}): J_{\mathrm{AH}}=\sum_{n=1}^{N} A_{n} / C_{n} ; \\
& \text { energy hypothesis }(\mathrm{EH}): J_{\mathrm{EH}}=1 / 2 \cdot \sum_{n=1}^{N}\left(A_{n} / C_{n}\right)^{2} ;
\end{aligned}
$$

pressure hypothesis $(\mathrm{PH}): J_{\mathrm{PH}}=\langle|\tilde{S}(t)|\rangle$;

where $\tilde{S}(t)$ is the filtered signal from Equation 2, $|x|$ denotes the absolute value of $x$, and $\langle y(t)\rangle$ is the temporal average of $y(t)$.

(3) The average firing rate $r$ is determined according to a single nonlinear function $r(J)$.

Note that the effective sound intensity $J$ as defined above is distinct from the physical sound intensity, commonly measured in decibels SPL (compare Eq. 21 in the Appendix), which we denote by $I$ throughout the text. Whereas $I$ measures the stimulus itself, $J$ is a derived quantity that incorporates the filter constants $C_{n}$ and therefore also reflects the sensitivity of the specific receptor cell. Furthermore, $I$ is defined as a logarithmic measure (relative to a predefined reference intensity); $J$ is not, which facilitates the notation.

Within the model framework, the filter constants $C_{n}$ are determined only up to a common factor, which can be absorbed in the function $r(J)$. In other words, the model remains unchanged if all $C_{n}$ are multiplied by the same constant and $r(J)$ is at the same time adjusted appropriately. It follows that one way to determine the $C_{n}$ is to choose a fixed firing rate, find for each frequency $f_{n}$ the amplitude $\hat{A}_{n}$ that leads to this firing rate, and set $C_{n}=\hat{A}_{n}$

In the following description of the experimental procedure, we will for simplicity focus on the case of superpositions of two tones. The generalization of concepts and formulas to the three-tone case is straightforward.

The three alternative hypotheses result in different predictions about which combinations of amplitudes $\left(A_{1}, A_{2}\right)$ are expected to lead to the same firing rate. Because the model implies that equal firing rate follows from equal effective sound intensity $J$ (step 3 ), curves of constant firing rate can easily be calculated for each hypothesis by setting $J$ constant in the equations of the second step in the model. These "iso-firing-rate curves" are shown in Figure 2. From the amplitude hypothesis, pairs $\left(A_{1}\right.$, $A_{2}$ ) yielding the same firing rate are expected to lie on a straight line. Likewise, from the energy hypothesis, they are expected to lie on an ellipse. For the pressure hypothesis, they should fall on an even more

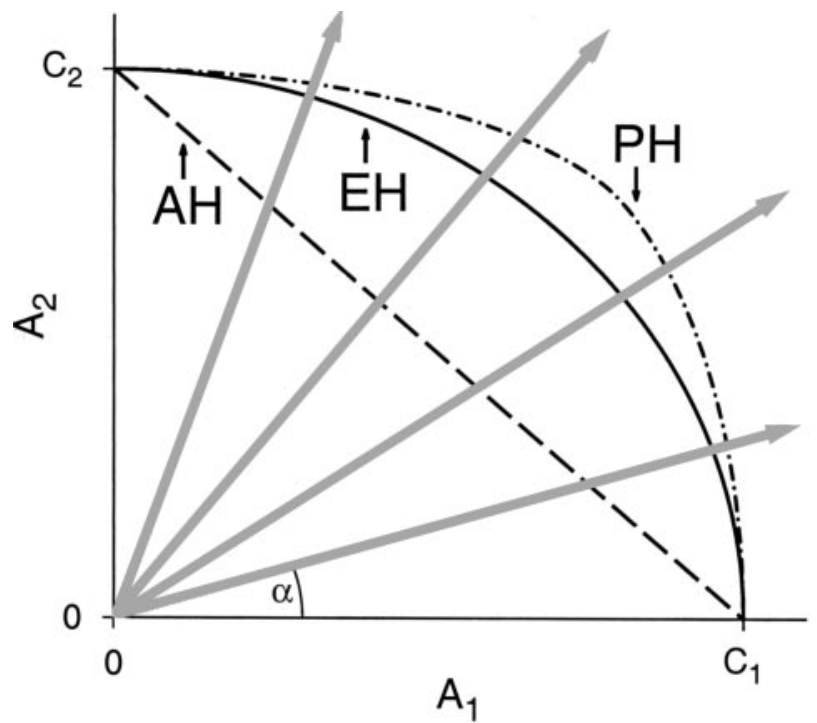

Figure 2. Prediction of iso-firing-rate curves for the superposition of two pure tones. Depending on the model, the effective sound intensity $J$ as well as the firing rate are expected to be constant along different curves in the two-dimensional space of amplitude combinations. $A_{1}$ and $A_{2}$ denote the amplitudes of the respective components. According to the amplitude hypothesis $(A H)$, iso-firing-rate curves are straight lines (one example shown by the dashed line); according to the energy hypothesis $(E H)$, they are ellipses (solid line); and according to the pressure hypothesis $(P H)$, they are even more strongly bent curves (dash-dotted line), the exact shape of which has to be determined numerically. The scale of the axes is given by the filter constants $C_{1}$ and $C_{2}$. Note that when the hypotheses are fitted to the data, the obtained filter constants will in general be different for each model, and the intersection points with the axes will not coincide because $C_{1}$ and $C_{2}$ are free parameters for each model. The gray arrows indicate equally spaced directions along which the rate-intensity curves are measured. In each direction, the intensity increases with increasing amplitudes $A_{1}$ and $A_{2}$, whereas $A_{1} / A_{2}$ is kept fixed and determined by the angle $\alpha$. (One example for this angle is denoted in the figure.) The intersection points of the arrows with the iso-firing-rate curves denote the amplitude combinations that are expected to yield the specified firing rate according to each of the three alternative hypotheses. Because the three intersection points on each gray arrow clearly differ from each other, the measurements of the iso-firing-rate curves can be used to distinguish between the hypotheses.

strongly bent curve. The corresponding shape has to be computed numerically by solving the equation:

$$
\langle|\tilde{S}(t)|\rangle=\frac{1}{\tau} \int_{0}^{\tau} d t\left|\frac{A_{1}}{C_{1}} \sin \left(2 \pi f_{1} t\right)+\frac{A_{2}}{C_{2}} \sin \left(2 \pi f_{2} t\right)\right|=\text { constant },
$$

for pairs:

$$
\left(\frac{A_{1}}{C_{1}}, \frac{A_{2}}{C_{2}}\right)
$$

The duration $\tau$ has to be chosen large enough to cover many cycles of the sine waves in the signal, so that the phases $\tilde{\varphi}_{n}$ can be neglected. Note that the shape of these three alternative iso-firing-rate curves is not influenced in any way by the form of $r(J)$.

To relate these predictions to experimental results, we determined a set of amplitude combinations leading to the same average firing rate in the following way. We start by measuring a first rate-intensity function for a single pure tone with frequency $f_{1}$. From this rate-intensity function, we determine the amplitude $A_{1}^{(1)}$ that leads to a firing rate of, e.g., $150 \mathrm{~Hz}$ as shown in Figure 1. (In the notation $A_{i}^{(n)}$, the subscript $i$ refers to the frequency $f_{i}$ at which the amplitude is measured, and the superscript $n$ indicates the number of the measurement, $1 \leq n \leq N$ where $N$ denotes the total number of measurements.) Because the amplitude $A_{2}$ of the second frequency component is zero for this stimulus, we denote the result as a data point $\left(A_{1}^{(1)}, 0\right)$, i.e., a point on the $A_{1}$ axis in a graph such 
as that of Figure 2. The same procedure is performed for a pure tone with frequency $f_{2}$, leading to a second data point $\left(0, A_{2}^{(2)}\right)$ on the $A_{2}$ axis that also corresponds to a firing rate of $150 \mathrm{~Hz}$. These two amplitudes $A_{1}^{(1)}$ and $A_{2}^{(2)}$ can already serve as estimates of the filter constants $C_{1}$ and $C_{2}$, respectively. We proceed by measuring rate-intensity functions for superpositions of the two tones where the ratio of the amplitudes $A_{1}$ and $A_{2}$ is held fixed. To do so, we set $A_{1}=k \cdot A_{2}$ and then jointly vary the intensity of $A_{1}$ and $A_{2}$. This corresponds to measuring the rate-intensity functions along straight lines in radial direction as pictured by the gray arrows in Figure 2. It is also evident from the figure that the radial direction is well suited for accurate measurements of the iso-firing-rate curves and for discriminating between the hypotheses. The resulting rate-intensity functions are similar in shape to the ones for the pure tones, and we can again determine the stimulus that leads to a firing rate of $150 \mathrm{~Hz}$ as in Figure 1 . This yields a third data point $\left(A_{1}^{(3)}, A_{2}^{(3)}\right)$ with $A_{1}^{(3)} / A_{2}^{(3)}=k$. The procedure is continued for several different ratios $k$ so that a set of amplitude pairs $\left(A_{1}^{(n)}, A_{2}^{(n)}\right)$ is obtained.

A technical but important question is which ratios $k$ should be used in the experiment. If the neuron is much more sensitive to one of the sound frequencies and if both amplitudes are comparable in size, i.e., $A_{1} \approx A_{2}$, the response will be determined almost exclusively by the more effective sound frequency. To be most informative, the measurement should thus take the relative sensitivities into account. This is done by choosing $k$ so that $A_{1} / C_{1}$ and $A_{2} / C_{2}$ are of the same order of magnitude, which assures that the effect of both tones is roughly the same. To do so, we use the estimates of $C_{1}$ and $C_{2}$ that have been obtained from the first two rate-intensity functions for the pure tones as explained above. In particular, the different ratios of $A_{1}$ and $A_{2}$ for subsequent measurements are selected on-line in such a way that after taking $C_{1}$ and $C_{2}$ into account, the directions along which the rate-intensity functions are measured are evenly spaced. The gray arrows in Figure 2 are such directions. Note that their even spacing depends on the scales of the axes given by $C_{1}$ and $C_{2}$. The calculation that achieves this is as follows: choose angles $\alpha$ that are evenly spaced in the interval $\left[0^{\circ}, 90^{\circ}\right]$ and use the relation for the slope $\rho$ of a straight line:

$$
\rho=\tan \alpha=\frac{A_{1} / C_{1}}{A_{2} / C_{2}}=k \cdot \frac{C_{2}}{C_{1}} .
$$

In the off-line analysis, the parameters $C_{1}$ and $C_{2}$ were newly determined by $\chi^{2}$ fits of each of the three curves in Figure 2 to the complete data set $\left(A_{1}^{(n)}, A_{2}^{(n)}\right)$. These fitted values of $C_{1}$ and $C_{2}$ should be more reliable than the initial estimates, which were obtained on-line from the pure-tone rate-intensity functions only.

A further technical detail concerns the choice of the fitting procedure. The procedure should treat $A_{1}$ and $A_{2}$ in a symmetric fashion, and it should not be affected by potentially large differences in the relative sensitivities for the two tones. This discards, e.g., the simplest choice of regarding $A_{2}$ as a function of $A_{1}$ or vice versa. Instead, we normalized the amplitudes by the filter constants and looked at the radial distance of the data points:

$$
\left(\frac{A_{1}^{(n)}}{C_{1}}, \frac{A_{2}^{(n)}}{C_{2}}\right),
$$

from the origin, which is given by:

$$
\sqrt{\left(\frac{A_{1}^{(n)}}{C_{1}}\right)^{2}+\left(\frac{A_{2}^{(n)}}{C_{2}}\right)^{2}}
$$

as a function of the ratio:

$$
\rho_{n}=\frac{A_{1}^{(n)} / C_{1}}{A_{2}^{(n)} / C_{2}} .
$$

This is a natural choice because the rate-intensity functions that led to the data points were measured in this radial direction. For the three hypotheses, we denote the predicted radial distance by $d_{m}^{(n)}$, where $m$ stands for the particular model hypothesis ( $m=\mathrm{AH}, \mathrm{EH}$, or $\mathrm{PH}) . d_{m}^{(n)}$ can be obtained from the model as a function of $\rho_{n}$ and corresponds to the normalized distance from the origin to the respective iso-firing-rate curve in Figure 2. For the amplitude hypothesis, one obtains:

$$
d_{\mathrm{AH}}^{(n)}=\sqrt{\rho_{n}^{2}+1} /\left(\rho_{n}+1\right),
$$

for the energy hypothesis, $d_{\mathrm{EH}}^{(n)}=1$, and for the pressure hypothesis, $d_{\mathrm{PH}}^{(n)}$ has to be determined numerically using the solutions of Equation 3.
Estimating $C_{1}$ and $C_{2}$ then corresponds to minimizing the $\chi^{2}$ function for the radial distance for each model $\mathrm{m}$ :

$$
\chi_{m}^{2}\left(C_{1}, C_{2}\right)=\sum_{n} \frac{\left[\sqrt{\left(\frac{A_{1}^{(n)}}{C_{1}}\right)^{2}+\left(\frac{A_{2}^{(n)}}{C_{2}}\right)^{2}}-d_{m}^{(n)}\right]^{2}}{\sigma_{n}^{2}},
$$

with respect to $C_{1}$ and $C_{2}$. The contributions of the data points are weighted by the measurement errors $\sigma_{n}$, which follow from the measurement errors $\Delta A_{1}^{(n)}$ and $\Delta A_{2}^{(n)}$ for $A_{1}^{(n)}$ and $A_{2}^{(n)}$, respectively, by the law of error propagation as:

$$
\sigma_{n}=\sqrt{\frac{\left(\frac{A_{1}^{(n)} \cdot \Delta A_{1}^{(n)}}{C_{1}^{2}}\right)^{2}+\left(\frac{A_{2}^{(n)} \cdot \Delta A_{2}^{(n)}}{C_{2}^{2}}\right)^{2}}{\left(\frac{A_{1}^{(n)}}{C_{1}}\right)^{2}+\left(\frac{A_{2}^{(n)}}{C_{2}}\right)^{2}}} .
$$

The fitted curves and the $\chi^{2}$ values obtained from the fits were used for further statistical analysis (see below).

For the control experiments with stimulus lengths of 300 or $500 \mathrm{msec}$, the onset response and the steady-state response were analyzed individually. For the onset, only spikes in the first $30 \mathrm{msec}$ after stimulus onset were taken into account; for the steady state, the first $200 \mathrm{msec}$ of the response were disregarded. The control experiments were aimed at investigating the effect of adaptation on our model description. We therefore performed the same analysis as explained above on the firing rates obtained for the onset and the steady state and fitted the filter constants $C_{1}$ and $C_{2}$ separately in each case. In addition, we compared $C_{1}$ and $C_{2}$ as well as their ratio $R=C_{1} / C_{2}$ for the onset with the respective values for the steady state. The relative change of $R$ was computed from the onset value, $R_{\mathrm{O}}$, and the steady-state value, $R_{\mathrm{S}}$, as $\Delta R=\left|R_{\mathrm{O}}-R_{\mathrm{S}}\right| / R_{\mathrm{S}}$. For the total response, the ratio of $C_{1}$ and $C_{2}$ is denoted by $R_{\text {total }}$. To estimate the significance of changes in $C_{1}, C_{2}$, and $R$ between the onset and the steady state, error measures for these parameters were computed for each cell individually by taking several nonoverlapping stretches of 30 msec during the steady state for the analysis, determining $C_{1}, C_{2}$, and $R$ in each case, and computing the respective SDs.

Experiments with superpositions of three pure tones were performed and analyzed in the same way as the two-tone experiments. We first measured rate-intensity functions for each pure tone and from these obtained initial estimates of the respective filter constants $C_{1}, C_{2}$, and $C_{3}$. Subsequently, rate-intensity functions were measured along different directions in the three-dimensional stimulus space:

$$
\left(\frac{A_{1}}{C_{1}}, \frac{A_{2}}{C_{2}}, \frac{A_{3}}{C_{3}}\right)
$$

The ratios of:

$$
\frac{A_{1}}{C_{1}}: \frac{A_{2}}{C_{2}}: \frac{A_{3}}{C_{3}},
$$

were taken as 1:1:1, 2:1:1, 1:2:1, and 1:1:2. Final fits of the model parameters $C_{1}, C_{2}$, and $C_{3}$ were obtained in an analogous way as for the superposition of two tones.

Statistical analysis. The $\chi^{2}$ values obtained from the fits were used to test the statistical significance of deviations of the data from the models by a standard $\chi^{2}$ test for each cell individually.

The Bayesian probability of a model given the data can be used as a measure for the preference of one hypothesis over another. It is calculated from Bayes' formula:

$$
p(\text { model } m \mid \text { data })=\frac{p(\text { data } \mid \text { model } m)}{p(\text { data })} \cdot p(\operatorname{model} m),
$$

where $p($ data $)=\Sigma_{m} p($ data $\mid \operatorname{model} m) \cdot p(\operatorname{model} m)$. If there is no a priori evidence for any model, the prior probabilities for the models are to be set to $p($ model $m)=1 / M$, where $M$ is the number of models investigated. 
The probabilities $p$ (data $\mid$ model $m$ ) were calculated from the difference between:

$$
\sqrt{\left(\frac{A_{1}^{(n)}}{C_{1}}\right)^{2}+\left(\frac{A_{2}^{(n)}}{C_{2}}\right)^{2}}
$$

and the corresponding model predictions $d_{m}^{(n)}$ by assuming independent errors with a Gaussian distribution of SDs $\sigma_{n}$ (given by the measurement errors) and a finite and fixed measurement resolution $\Delta$ :

$p($ data $\mid \operatorname{model} m)=$

$$
\Delta \cdot \prod_{n} \frac{1}{\sqrt{2 \pi \sigma_{n}^{2}}} \exp \left\{-\frac{1}{2 \sigma_{n}^{2}} \cdot\left[\sqrt{\left(\frac{A_{1}^{(n)}}{C_{1}}\right)^{2}+\left(\frac{A_{2}^{(n)}}{C_{2}}\right)^{2}}-d_{m}^{(n)}\right]^{2}\right\} .
$$

An analogous formula was used in the case of superpositions of three pure tones.

Trends in the data were tested for statistical significance by a standard run test (Barlow, 1989). For a given model, the data points were subdivided into those sequences of points that lie consecutively either above or below the model prediction, and the number of these sequences was tested for significant deviations from the null hypothesis of independently scattered data points around the model prediction.

Comparison of pure-tone and noise stimuli. In another set of experiments, we tested whether our understanding of spectral integration allows accurate predictions of firing rates for more complex stimuli and focused on bandpass-filtered noise. To calibrate the model for a specific receptor, we measured the rate-intensity function for a pure tone as well as a set of filter constants in the relevant frequency band of the noise stimulus. According to our model, we can use these pure-tone results to calculate a prediction for the rate-intensity function of the noise stimulus (see below). The filter constants are needed for the calculation of the effective sound intensity $J$ for the noise stimulus (model step 2), and the pure-tone rate-intensity function is needed because it implicitly contains the information about the shape of the response function $r(J)$ of model step 3. To assess the reliability of the prediction, the rate-intensity function for the noise stimulus was also measured experimentally. The particular noise stimulus that we used was Gaussian white noise, cut off at $\pm 3 \mathrm{SDs}$ and bandpass filtered between 5 and $10 \mathrm{kHz}$, and the frequency of the pure-tone stimulus was $4 \mathrm{kHz}$. Note that when the amplitude of a noise stimulus is varied, all amplitudes in the signal are scaled by a common factor.

The prediction for the rate-intensity function of the noise signal is obtained in the following way. According to our model, the rate-intensity function of the pure tone, $r^{\mathrm{pt}}(I)$, and the rate-intensity function of the noise stimulus, $r^{\text {noise }}(I)$, should have the same shape and be related to each other by a shift $\Delta I$ along the decibel-intensity axis:

$$
r^{\text {noise }}(I)=r^{\mathrm{pt}}(I-\Delta I) .
$$

For notational simplicity, we always use the same symbol $r$ to denote the firing rate regardless of whether we consider its dependence on the sound intensity $I, r(I)$, or on the effective sound intensity $J, r(J)$. Strictly speaking, $r(I)$ and $r(J)$ are different functions, but from the context, it will always be clear to which function we refer.

Let us briefly describe the reason for the relation of Equation 8. For concreteness, we focus on the energy hypothesis; the amplitude and the pressure hypotheses can be dealt with in an analogous way. Consider an arbitrary sound signal $S(t)$ composed of a set of pure tones with amplitudes $A_{n}$. From these, we can calculate the intensity, which is defined as:

$$
I=20 \log _{10} \frac{\sqrt{\left\langle S(t)^{2}\right\rangle}}{20 \mu \mathrm{Pa}}=20 \log _{10} \frac{\sqrt{\frac{1}{2} \cdot \sum_{n} A_{n}{ }^{2}}}{20 \mu \mathrm{Pa}},
$$

as well as the effective sound intensity:

$$
J_{\mathrm{EH}}=\frac{1}{2} \Sigma_{n}\left(\frac{A_{n}}{C_{n}}\right)^{2} .
$$

The essential observation is that multiplying every $A_{n}$ by the same factor $k$ amounts to adding a constant $20 \log _{10} k$ to the intensity $I$ (if $k<1$, this constant is negative), whereas $J_{\mathrm{EH}}$ is multiplied by a factor $k^{2}$.

We now consider a noise stimulus with intensity $I^{\text {noise }}$ and effective sound intensity $J_{\mathrm{EH}}^{\text {noise }}$. To compare the response with that of a pure tone, we find the intensity $I^{\mathrm{pt}}$ that yields the same firing rate as the noise stimulus by setting both effective sound intensities equal:

$$
J_{\mathrm{EH}}^{\mathrm{pt}}=\frac{1}{2}\left(\frac{A^{p t}}{C^{p t}}\right)^{2}=J_{\mathrm{EH}}^{\mathrm{noise}} .
$$

The parameter $C^{\mathrm{pt}}$ denotes the filter constant for the pure tone. From the preceding equation, we can calculate the pure-tone amplitude $A^{\mathrm{pt}}$ and thus the intensity $I^{\mathrm{pt}}$ of the pure tone, for which the firing rate is the same as for the noise signal with given intensity $I^{\text {noise }}$. Let us denote the difference between $I^{\text {noise }}$ and $I^{\mathrm{pt}}$ by $\Delta I$.

If we multiply all amplitudes by the same factor $k$, the amplitudes of the noise signal as well as $A^{\mathrm{pt}}$, the intensities $I^{\text {noise }}$ and $I^{\mathrm{pt}}$ are changed by the same amount. Consequently, the difference between the new intensities is still given by $\Delta I$. Likewise, the effective sound intensities are multiplied by the same factor, i.e., we still have $J_{\mathrm{EH}}^{\mathrm{pt}}=J_{\mathrm{EH}}^{\text {noise }}$. Because the firing rate depends only on the value of $J$, this means that for the new intensities, the firing rates are also equal. It follows that whenever the intensities of the pure tone and the noise signal differ by $\Delta I$, the firing rates for the two stimuli are the same. A thorough mathematical derivation of this concept, which also yields explicit expressions for the amount of the shifts for the energy and pressure hypotheses, can be found in the Appendix.

The derivation shows that the predicted $\Delta I$ is given by:

$$
\Delta I_{\mathrm{EH}}=-10 \log _{10}\left(\left(C^{\mathrm{pt}}\right)^{2} \frac{\sum_{n} A_{n}^{2} / C_{n}^{2}}{\sum_{n} A_{n}^{2}}\right)
$$

for the energy hypothesis and by:

$$
\Delta I_{\mathrm{PH}}=-10 \log _{10}\left(\frac{\pi}{4}\left(C^{\mathrm{pt}}\right)^{2} \frac{\sum_{n} A_{n}^{2} / C_{n}^{2}}{\sum_{n} A_{n}^{2}}\right),
$$

for the pressure hypothesis. The two predictions for $\Delta I$ differ by $-10 \log _{10}$ $\frac{\pi}{4} \approx 1.05 \mathrm{~dB}$. Because this is below our measurement accuracy, we do not use this experiment for distinguishing between the hypotheses, but rather as a test of the generality and the predictive power of the model per se.

Evaluating Equations 10 and 11 is possible if one knows the filter constants and the $A_{n}^{2}$ for the amplitudes in the noise signal. The latter are given by the power spectrum of the noise signal, which we calculated in discretized bins of width $0.05 \mathrm{kHz}$ (using a triangular Bartlett window). Filter constants $C_{n}$ were measured for pure tones between 5 and $10 \mathrm{kHz}$ at every $0.2-1 \mathrm{kHz}$ (depending on the length of the recording) by determining the amplitude that led to a firing rate of $260 \mathrm{~Hz}$. Additional filter constants $C_{n}$, for all center frequencies of the power-spectrum bins, can be determined by linear interpolation from the measured filter constants.

The prediction for the noise-stimulus rate-intensity function that results from shifting the pure-tone rate-intensity function $r^{\mathrm{pt}}(I)$ by $\Delta I$ is compared with the measured curve $r^{\text {noise }}(I)$. To do this quantitatively, the prediction of $\Delta I$ is related to the true shift $\Delta I_{\text {true }}$ that can be extracted from the measured rate-intensity functions of the pure tone and the noise signal as the distance between these two functions. Because the rateintensity functions are given by individual pairs of intensity and firing rate, $(I, r)$, we use the distance of such a data point of one rate-intensity function to the approximate location of the other rate-intensity function. For a data point $\left(I^{\mathrm{pt}}, r^{\mathrm{pt}}\right)$ from pure-tone stimulation, e.g., we thus determine the intensity $\hat{I}^{\text {noise }}$ that would be expected to lead to the same firing rate $r^{\mathrm{pt}}$, but for the noise stimulus. The determination of $\hat{I}^{\text {noise }}$ given the firing rate $r^{\mathrm{pt}}$ is again done by linear interpolation of the noise rate-intensity function as in Figure 1 . We thus find for every intensity $I_{n}^{\mathrm{pt}}$ of the pure-tone rate-intensity function a corresponding $\hat{I}_{n}^{\text {noise }}$, and similarly for every intensity $I_{m}^{\text {noise }}$ of the noise rate-intensity function a corresponding $\hat{I}_{m}^{\mathrm{pt}}$. Because ideally, these should be related by $\hat{I}_{n}^{\text {noise }}=$ $I_{n}^{\mathrm{pt}}+\Delta I_{\text {true }}$ and $\hat{I}_{m}^{\mathrm{pt}}=I_{m}^{\text {noise }}-\Delta I_{\text {true }}$, we can estimate $\Delta I_{\text {true }}$ by minimizing the $\chi^{2}$ function:

$$
\chi^{2}\left(\Delta I_{\text {true }}\right)=\sum_{n}\left(I_{n}^{\mathrm{pt}}-\hat{I}_{n}^{\text {noise }}+\Delta I_{\text {true }}\right)^{2}+\sum_{m}\left(I_{m}^{\text {noise }}-\hat{I}_{m}^{\mathrm{pt}}-\Delta I_{\text {true }}\right)^{2} .
$$

Because the subthreshold part and the saturation are not important in the determination of the actual shift, only data points $(I, r)$ with $r$ between 20 and $80 \%$ of the maximum firing rate of the cell were taken into account. 

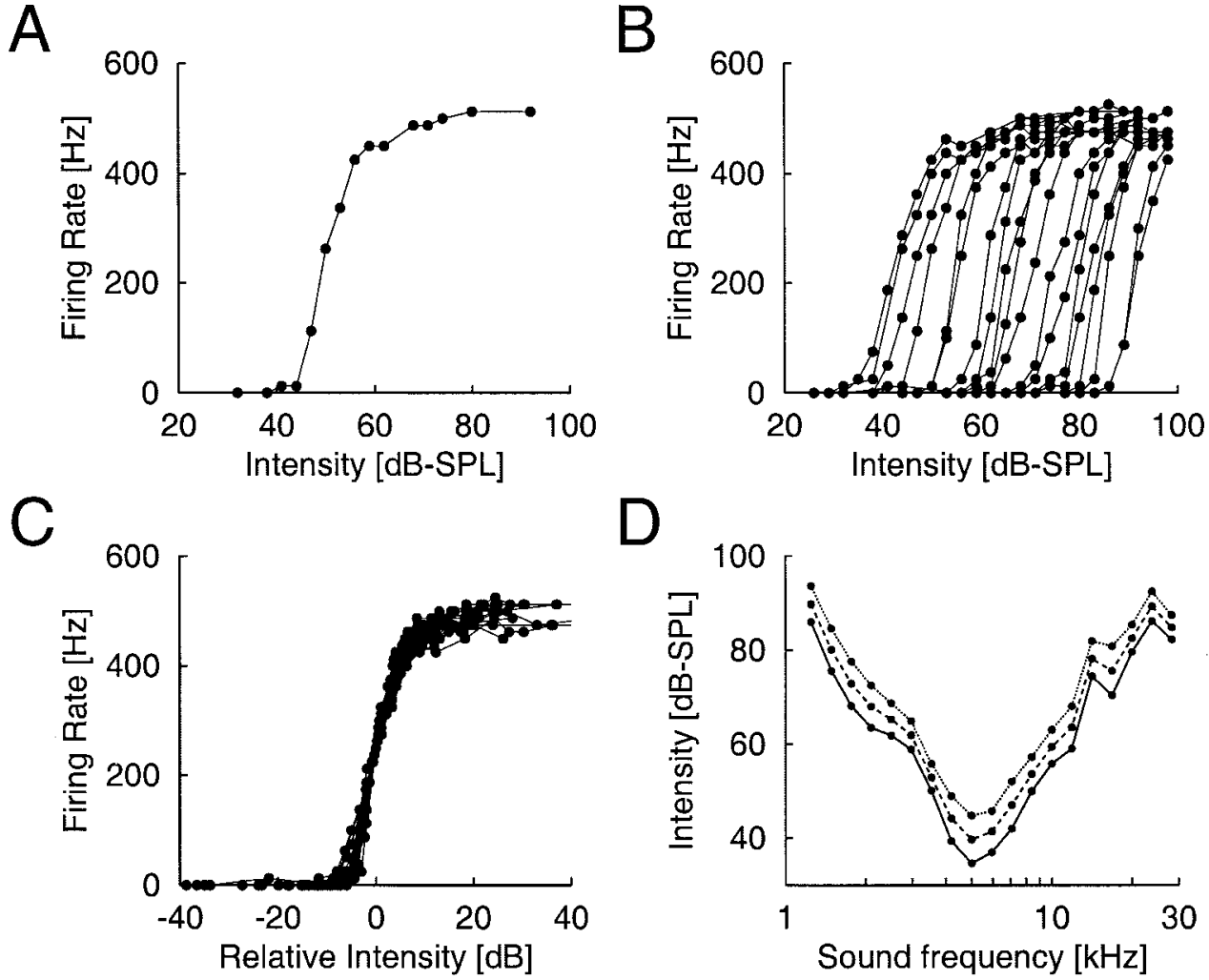

Figure 3. Firing-rate responses of a locust auditory receptor cell. A, Rateintensity function for a $7 \mathrm{kHz}$ pure tone. The observed sigmoidal shape of the rate-intensity function is typical for many receptor types. Below a threshold of $\sim 45 \mathrm{~dB}$ SPL, the cell shows virtually no response. $B$, Rate-intensity functions of the same neuron for many different pure tones between 1.25 and $28 \mathrm{kHz}$. Connected points belong to the same sound frequency. Curves farther to the left correspond to frequencies at which the cell is more sensitive. Although there are large differences concerning the intensity range where the individual rate-intensity functions rise from threshold to saturation, their overall shape is very similar. For example, all measured rate-intensity functions have approximately the same slope in the rising part of the curves and saturate at around the same level. $C$, The same rate-intensity functions as in $B$, now shifted along the decibel axis such that they align at a firing rate of $250 \mathrm{~Hz}$. This demonstrates the generic shape of the rate-intensity functions. $D$, Curves denoting equal firing rates at different sound intensities for the same cell. The threshold curve (solid line) and the intensities corresponding to constant firing rates of $150 \mathrm{~Hz}$ (dashed line) and 300 $\mathrm{Hz}$ (dotted line) are shown for pure tones between 1.25 and $28 \mathrm{kHz}$. The three curves are approximately parallel to each other, reflecting the similarity of the rate-intensity functions for different frequencies.

\section{RESULTS}

The objective of this study is to develop a descriptive model for the responses of auditory receptor neurons to arbitrary stationary acoustic stimuli. This is done to identify the dominant physical stimulus property governing the encoding of sound intensity. We first develop a general mathematical framework for the transformation of the incoming sound into the neural response. Subsequently, we apply the model to locust auditory receptors and show that the experimental data are well described by only one of three rival hypotheses about the nature of the primary signal transduction.

\section{Derivation of the mathematical model}

In locusts, auditory signals are encoded by $60-80$ receptor neurons at each ear with similar general properties but considerable variability in the parameter values describing the sensitivity of individual neurons to specific sound frequencies (Römer, 1985). In response to a pure tone with sufficient intensity, the firing rate of a receptor cell increases in a sigmoidal fashion with stimulus intensity (Fig. $3 A$ ). The steepness and level of saturation of this rate-intensity function depend on the individual cell and temperature. Below a threshold intensity, there is no or only very low spontaneous activity. The regime between threshold and saturation usually spans $\sim 15-30 \mathrm{~dB}$, and maximum firing rates lie at $\sim 300 \mathrm{~Hz}$ for room temperature and $\sim 500 \mathrm{~Hz}$ for $30^{\circ} \mathrm{C}$.

The frequency-resolved sensitivity of the receptors can be characterized by a threshold curve, i.e., the dependence of the threshold on the sound frequency (Fig. 3D). The receptors are fairly broadly tuned with characteristic frequencies in the range of $4 \mathrm{kHz}$ (low-frequency receptors) to $15 \mathrm{kHz}$ (high-frequency receptors), and the absolute sensitivities vary strongly between individual neurons (Römer, 1985; Jacobs et al., 1999).

Measuring rate-intensity functions from a single receptor cell for many different sound frequencies reveals another property of the receptors; to good approximation, the rate-intensity functions are shifted versions of one another along the intensity axis, where intensity is measured in the logarithmic units of sound-pressure level, decibels SPL. This phenomenon has been reported previously by Suga (1960) and Römer (1976). A detailed example with frequencies spanning the whole sensitivity range of a typical low-frequency receptor (characteristic frequency of $\approx 5 \mathrm{kHz}$ ) can be seen in Figure $3 B$. The generic shape of the rate-intensity functions becomes even clearer if they are shifted relative to each other and aligned at $250 \mathrm{~Hz}$ firing rate (Fig. $3 C$ ). Figure $3 D$ shows the threshold curve together with curves denoting the intensities that lead to firing rates of 150 and $300 \mathrm{~Hz}$. As a consequence of the generic shape of the rate-intensity functions, all curves are approximately parallel to each other.

These key findings indicate that over the whole frequency range, the coupling of the physical stimulus is not substantially influenced by mechanical nonlinearities. In fact, a simple filtering mechanism captures the essence of the observed phenomenon. Let us assume that for all pure tones the firing rate is given by a single function $r\left(A_{n} / C_{n}\right) . A_{n}$ denotes the amplitude of a specific pure tone of frequency $f_{n}$, and $C_{n}$ is a frequency-dependent filter constant such that the firing rate depends only on the ratio $A_{n} / C_{n}$. This corresponds to a gain factor of $1 / C_{n}$ for each sound frequency. For two different frequencies $f_{1}$ and $f_{2}$, the firing rates $r\left(A_{1} / C_{1}\right)$ and $r\left(A_{2} / C_{2}\right)$ are then the same when $A_{1} / C_{1}=A_{2} / C_{2}$, i.e., when the amplitudes take on a constant ratio $A_{1} / A_{2}=C_{1} / C_{2}$. 
Because the intensity $I$ in decibels SPL is defined as a logarithmic measure of the amplitude, $I=20 \log _{10}(A /(\sqrt{2} \cdot 20 \mu \mathrm{Pa}))$, this constant amplitude ratio corresponds to a constant intensity difference, $\Delta I=I_{1}-I_{2}=20 \log _{10}\left(C_{1} / C_{2}\right)$. The firing rates for the two tones are therefore always the same if their intensities differ by $\Delta I$. The rate-intensity functions are thus shifted versions of one another separated by $\Delta I$ as found in the experiment. Generalizing this idea to stimuli containing more than one frequency leads us to the first step of our model:

\section{Step 1: coupling to the stimulus}

The sound pressure wave $S(t)$, written as a Fourier series:

$$
S(t)=\sum_{n=1}^{N} A_{n} \sin \left(2 \pi f_{n} t+\varphi_{n}\right),
$$

where the $f_{n}$ denote the frequencies, $\varphi_{n}$ phase offsets, and the $A_{n}$ the respective amplitudes, is initially transformed into a filtered signal $\tilde{S}(t)$ :

$$
\tilde{S}(t)=\sum_{n=1}^{N} \frac{A_{n}}{C_{n}} \sin \left(2 \pi f_{n} t+\tilde{\varphi}_{n}\right) .
$$

The amplitudes are multiplied by frequency-dependent gain factors $1 / C_{n}$. These describe the frequency-resolved sensitivity, i.e., the tuning of the receptor cell, and correspond directly to the values of the threshold curve at the frequencies $f_{n}$. In addition, a putative phase shift turns $\varphi_{n}$ into $\tilde{\varphi}_{n}$.

Although the above reasoning for using a linear filter as the first model step is based on electrophysiological observations only, it corresponds well with biophysical findings regarding the tympanal membrane. Schiolten et al. (1981) observed that the tympanal membrane behaves approximately as a linear oscillator with a short damping time constant of $\sim 100 \mu \mathrm{sec}$. The resonance properties of this oscillator are thought to be responsible for the frequency-resolved gain of the receptors and therefore also for the shapes of the threshold curves (Michelsen, 1971a, 1971b, 1979). Michelsen and Rohrseitz (1995) also note that the amplitude of the tympanal vibration depends linearly on the sound pressure for pure tones.

\section{Step 2: mechanosensory transduction}

Receptor cells are attached to the tympanal membrane with a cilium protruding from the dendrite and several auxiliary cells surrounding a receptor (Gray, 1960). The biophysical functioning of this machinery is not yet understood, but oscillations of the tympanal membrane presumably lead to conductance changes in the receptors' dendrites that give rise to membrane depolarizations (Hill, 1983a, 1983b). This is where a spectral integration of frequency-dependent stimulus attributes must occur. Voltage fluctuations in the range of the relevant sound frequencies (several kilohertz) cannot be transmitted by the cell membrane because of its low-pass filter properties. Information about the spectral content is therefore lost at the level of the membrane potential, which, instead, is expected to correspond to an integrated stimulus property. The spectrum of the generator potential after acoustic stimulation is indeed found to contain no trace of the sound frequency used (Hill, 1983a).

Following ideas from the literature concerning temporal integration in auditory receptor cells (Tougaard, 1996; Heil and Neubauer, 2001), we set up three hypotheses for the spectral integration by calculating an "effective sound intensity" $J$ from $\tilde{S}(t)$.
Amplitude hypothesis $(A H)$

$J$ corresponds to the maximum amplitude of $\tilde{S}(t)$. This is the common view of a threshold: a response occurs once the signal reaches a certain value. In the case of few frequency components, $J$ is given by the sum of the scaled amplitudes:

$$
J_{\mathrm{AH}}=\sum_{n=1}^{N} \frac{A_{n}}{C_{n}} .
$$

\section{Energy hypothesis (EH)}

$J$ corresponds to the temporal mean of the squared signal [throughout what follows, $\langle x(t)\rangle$ denotes the temporal mean of $x(t)]$ :

$$
J_{\mathrm{EH}}=\left\langle\tilde{S}(t)^{2}\right\rangle .
$$

From Parseval's Theorem (Press et al., 1992), we see that this expression can be rewritten as the sum of the squares of the scaled amplitudes:

$$
J_{\mathrm{EH}}=\frac{1}{2} \sum_{n=1}^{N} \frac{A_{n}^{2}}{C_{n}^{2}} .
$$

Because the square of the amplitude of a sinusoidal oscillation is proportional to the energy contained in the oscillation, this hypothesis reflects an energy-integration mechanism.

\section{Pressure hypothesis $(\mathrm{PH})$}

$J$ corresponds to the temporal mean of the absolute value of $\tilde{S}(t)$ :

$$
J_{\mathrm{PH}}=\langle|\tilde{S}(t)|\rangle .
$$

This hypothesis complies with a pressure-integration mechanism after half-wave rectification.

\section{Step 3: encoding by firing rates}

The response of an auditory receptor to a signal of constant intensity can be characterized by a mean firing rate $r$. The rate is obtained from a one-dimensional, nonlinear transformation of the effective sound intensity $J$ :

$$
\text { firing rate }=r(J)
$$

Note that the effective sound intensity $J$ is a theoretical construct, which does not necessarily correspond to a biophysical property. It is used here to describe regions of constant firing rate in stimulus space because these correspond to regions of constant $J$. Therefore, instead of the specifically simple versions of $J$ given above, we could also use any transformation $\tilde{J}=f(J)$ with some appropriate function $f$. This transformation does not affect the shape of the regions of constant $J$, but we can speculate that for the correct choice of $f, \tilde{J}$ has a direct biophysical interpretation, such as the change in membrane conductance caused by the stimulus.

Measured spike-train responses have a strong transient attributable to adaptation. In a first approach, we average over this temporal structure in the response and consider only the total number of spikes elicited by the stimulus. In a second, more detailed analysis, we analyze individual parts of the response to explicitly test how this structure in the spike trains might affect our model description. 

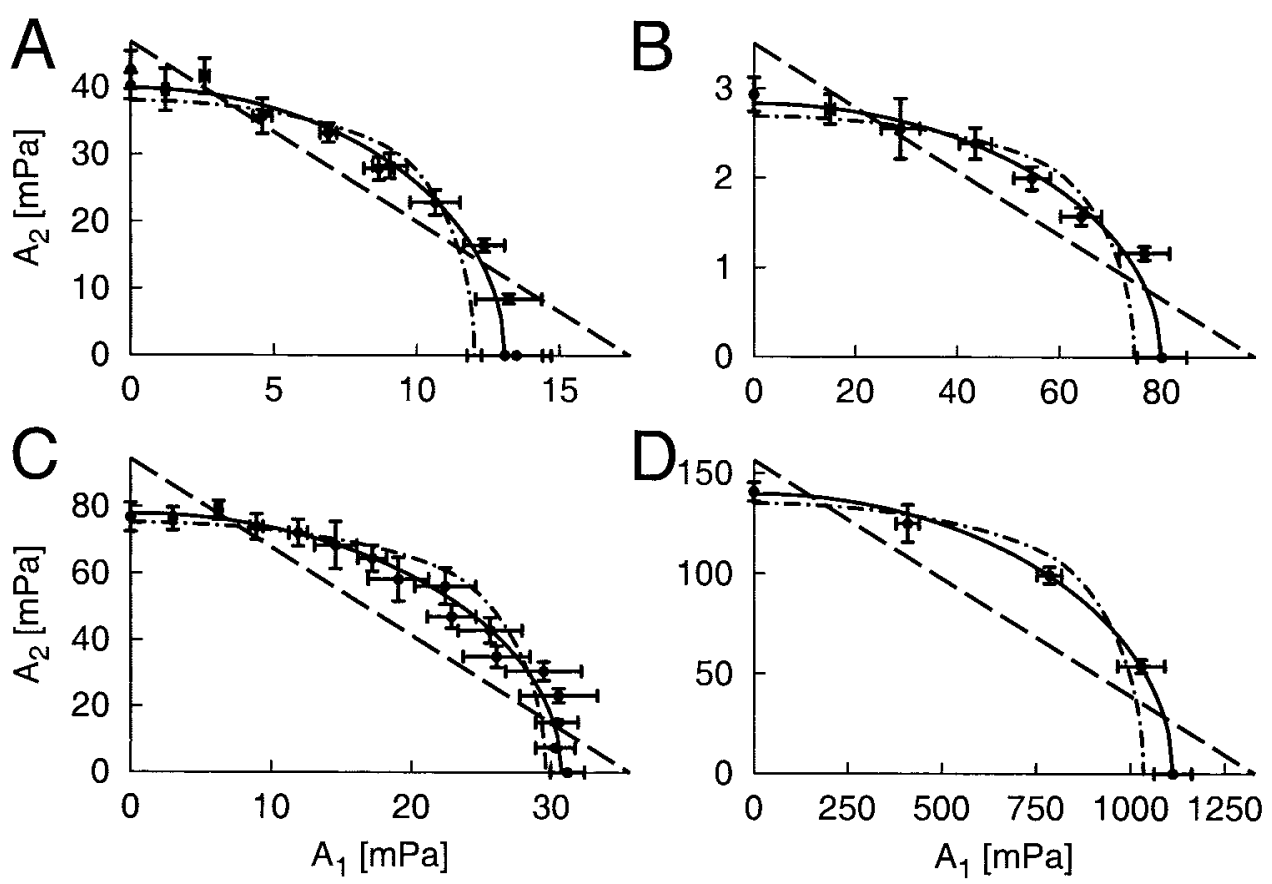

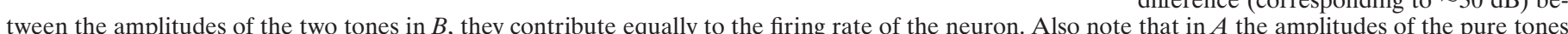
giving a firing rate of $150 \mathrm{~Hz}$ were measured twice (at the beginning and the end of the experiment), with the results approximately coinciding.

\section{Electrophysiological experiments}

\section{Experimental strategy}

To directly address the question of spectral integration and the hypotheses in step 2 of our model, we compare only stimuli that lead to the same firing rate of a given neuron. With this strategy, we circumvent complications attributable to the nonlinearity induced by the spike-generation mechanism. In terms of our model, a constant firing rate implies a constant effective sound intensity $J$ and vice versa, independently of the specific shape of $r(J)$. As a crucial element of our analysis, we therefore identify regions of constant $J$ in stimulus space $\left(A_{1}, A_{2}, \ldots\right)$ by searching for stimulus combinations that result in the same firing rate. We denote these regions as iso-firing-rate regions. They are then compared with the predictions of the three hypotheses and reveal how $J$ is composed of contributions from the single amplitudes. Because the rate-intensity functions are found to be fairly smooth in the rising part between threshold and saturation, extracting isofiring-rate regions can be accurately done by linear interpolation as is shown in Figure 1.

\section{Superpositions of two pure tones}

The complete stimulus space of stationary stimuli is, of course, high dimensional. We thus started with low-dimensional subspaces using only two or three pure tones and their superpositions. In the two-dimensional subspace $\left(A_{1}, A_{2}\right)$, each point represents a linear combination of two pure-tone signals at frequencies $f_{1}$ and $f_{2}$ (4 and $\left.9.55 \mathrm{kHz}\right)$ :

$$
S(t)=A_{1} \sin \left(2 \pi f_{1} t\right)+A_{2} \sin \left(2 \pi f_{2} t\right) .
$$

For these stimuli, we determined combinations $\left(A_{1}, A_{2}\right)$ that yield the same fixed firing rate. Figure 2 shows the shapes of the iso-firing-rate curves as predicted by the three hypotheses.

Responses to superpositions of two pure tones with stimulus duration of $100 \mathrm{msec}$ were measured for 17 cells. Figure 4 depicts sets of amplitude combinations $\left(A_{1}, A_{2}\right)$ that led to a firing rate of
Figure 4. Iso-firing-rate curves for superpositions of two pure tones for four different receptor cells $(A-D)$. The measured pairs of amplitudes corresponding to a firing rate of $150 \mathrm{~Hz}$ (small filled circles) are shown together with the isofiring-rate curves for the three hypotheses. For each curve, the two free parameters $C_{1}$ and $C_{2}$ were fitted to the data. The dashed lines denote the fits of the amplitude hypothesis, the solid lines denote the fits of the energy hypothesis, and the dash-dotted lines denote the fits of the pressure hypothesis. Although the curves for the amplitude and the pressure hypothesis deviate systematically, the ellipse obtained from the energy hypothesis corresponds well with the data. Note the different scales on the axes between the four cells as well as between the $x$-axis and the $y$-axis of individual plots. These differences are attributable to the strong dependence of the sensitivity on the sound frequency and the specific cell. From the fits of the energy hypothesis, we obtain the following ratios $C_{1} / C_{2}$ in these four cases: $0.33(A), 28.33(B), 0.39(C)$, and 7.96 $(D)$. Although there is an almost 30 -fold difference (corresponding to $\sim 30 \mathrm{~dB}$ ) be$150 \mathrm{~Hz}$ in each of the four cells presented. Fitted iso-firing-rate curves corresponding to the three hypotheses are also shown.

Performing a $\chi^{2}$ test on the fits of the three hypotheses showed that the amplitude hypothesis is rejected at the $1 \%$ level for all 17 cells, whereas the energy hypothesis is not rejected for any cell and the pressure hypothesis is rejected for 4 cells. For an in-depth analysis, we therefore considered only the energy and the pressure hypotheses.

To further distinguish between these two hypotheses, we directly compared the goodness of fit given by the $\chi^{2}$ values. The energy hypothesis yielded a lower $\chi^{2}$ than the pressure hypothesis in 16 of 17 cases. We also calculated a Bayesian estimate of the probability $p$ (model|data) of the model given the data (with prior probabilities of 0.5 for both the energy and the pressure hypothesis $)$. The mean of $p(\mathrm{EH} \mid$ data $)$ was obtained as 0.884 with 0.167 SD and median $0.978(N=17)$, whereas $p(\mathrm{PH} \mid$ data $)$ equals $1-$ $p(\mathrm{EH} \mid$ data $)$ and therefore had a mean of only 0.116 .

Furthermore, data points for which $A_{1} / C_{1}$ and $A_{2} / C_{2}$ were approximately equal (i.e., data points in the middle sections of the plots in Fig. 4) were in general below the fitted iso-firing-rate curve of the pressure hypothesis instead of scattered around it as would be expected if the deviations resulted from independent measurement errors. We investigated this trend by a run test for those fits of the model that had at least $10 \mathrm{df}$ (i.e., 12 data points). For these nine cells, the run test showed significant deviations $(p<0.01)$ from the pressure hypothesis in three cases. All three cells were different from those that had led to statistically significant deviations from the pressure hypothesis according to the $\chi^{2}$ test. For the energy hypothesis, such a trend was not observable.

From the combined evidence, we conclude that the amplitude as well as the pressure hypotheses can be rejected. The energy hypothesis, on the other hand, provides a good description of the data for spectral integration in the two-tone case.

The values obtained for the filter constants corresponding to the energy hypothesis can be read from the graphs in Figure 4 as 


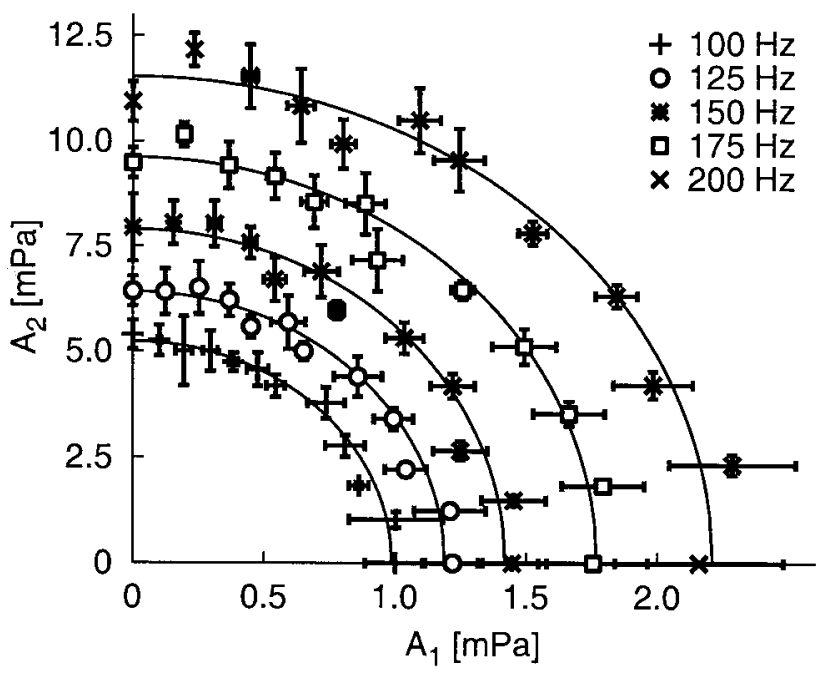

Figure 5. Iso-firing-rate curves for superpositions of two pure tones for one receptor cell at different firing rates. The points display measured pairs of amplitudes, and the solid lines are corresponding ellipses fitted to the data in accordance with the energy hypothesis. The firing rates rise from 100 to $200 \mathrm{~Hz}$ in steps of $25 \mathrm{~Hz}$. Note that the fits agree with the data regardless of the firing rate and that ellipses for different firing rates are scaled versions of each other as predicted by the energy hypothesis. The ratios of $C_{1} / C_{2}$ lie in the narrow range between 0.177 and 0.185 for all five firing rates.

the half-axes of the ellipses, i.e., as the intersection points of the ellipses with the two coordinate axes. Values range from $\sim 1$ to $2000 \mathrm{mPa}$, corresponding to the large variability in overall sensitivity of the receptors. As stated in Materials and Methods, the filter constants are determined only up to a common factor. Their ratios are, however, a direct measure of the relative sensitivity for the two chosen sound frequencies. In our experiments, we found ratios of $C_{1}$ and $C_{2}$ of up to $30: 1$ (Fig. $4 B$ ), which means that spectral integration can be accurately determined even if the sensitivities for the two sound frequencies diverge by as much as $30 \mathrm{~dB}$ and possibly more.

We also see from Figure 4 that the initial estimates of $C_{1}$ and $C_{2}$ (taken from the pure-tone rate-intensity functions; see Materials and Methods) are already very close to the values obtained by the fit of the energy hypothesis. The initial estimates for $C_{1}$ and $C_{2}$ are given by the data points on the coordinate axes and closely coincide with the intersection points of the ellipses. This shows that the filter constants measured with pure tones are approximately the same as those obtained from fitting the energy hypothesis to all data points.

As an additional test of the energy hypothesis, we investigated how iso-firing-rate curves that were obtained separately for different firing rates are related to one another. Figure 5 shows pairs $\left(A_{1}, A_{2}\right)$ corresponding to several firing rates between 100 and $200 \mathrm{~Hz}$. Pairs corresponding to the same firing rate are accurately fitted by ellipses. Each ellipse corresponds to an independent fit to the data points of the same firing rate. To good approximation, all ellipses are scaled versions of one another. This result is in accordance with the energy hypothesis, because the ratio of the half-axes of the ellipses should always equal the ratio of the filter constants $C_{1}$ and $C_{2}$. Such a behavior was observed for all cells measured. For each cell, we determined the ratios $R_{100}$ and $R_{150}$ of half-axes of the ellipses corresponding to 100 and $150 \mathrm{~Hz}$, respectively, and their relative deviations $\left|\left(R_{150}-R_{100}\right) / R_{150}\right|$.
We found that with a mean of 0.044 (SD 0.026), these were always small.

\section{Analysis of specific response episodes}

Up to now, we have disregarded the fact that the spike-train responses contain a pronounced transient attributable to adaptation. Typical spike trains from receptor cells for $300 \mathrm{msec}$ puretone stimuli and the corresponding instantaneous firing rates can be seen in Figure 6, $A$ and $E$. The transient usually spans approximately the first $40 \mathrm{msec}$ but can last as long as $100 \mathrm{msec}$. Afterward, the cell has adapted to the sound intensity, and the response is approximately in a stationary steady state for the rest of the stimulus duration. When the stimulus ends, the receptor cells do not show an offset response, but stop firing or return to their usually low spontaneous activity. To investigate how the transients influence our model description, we explicitly analyzed the validity of the hypotheses for the onset as well as the steadystate response.

Spike trains from 10 cells were recorded with stimuli of either $300 \mathrm{msec}$ (in 6 cases) or $500 \mathrm{msec}$ duration (in 4 cases). The same analysis as before was applied to the onset by using only the first $30 \mathrm{msec}$ of the response and to the steady state by disregarding the first $200 \mathrm{msec}$ after stimulus onset. Two examples are shown in Figure 6. In each case, the data points are best fitted by the ellipses from the energy hypothesis. We again performed a statistical analysis of the goodness of fit. Longer stimulus durations resulted in fewer measurements per cell so that the data points often had larger experimental errors. This effect is even stronger for the analysis of the onset response, which relies on considerably shorter stretches of data. Nevertheless, the data from two cells during steady state deviated significantly $(p<0.05)$ from the pressure hypothesis, whereas the energy hypothesis always gave a good fit. Furthermore, the Bayesian test favored the energy hypothesis over the pressure hypothesis strongly for both onset and steady state $[p(\mathrm{EH} \mid$ data $)$ for onset response: mean 0.642 with $0.100 \mathrm{SD}$, median $0.649 ; p(\mathrm{EH} \mid$ data $)$ for steady state: mean 0.795 with $0.131 \mathrm{SD}$, median $0.782 ; N=10]$. We conclude that the energy hypothesis yields an appropriate description also for the specific episodes of the response.

We may now use our description of spectral integration to investigate a possible dependence of the adaptation on the sound frequency. Adaptation mechanisms in mechanoreceptors have been identified for stimulus coupling (Eyzaguirre and Kuffler, 1955; Chapman et al., 1979), transduction (Ricci et al., 1998; Holt and Corey, 2000), and encoding (Matthews and Stein, 1969; Purali and Rydqvist, 1998). For insect mechanoreceptors, spike adaptation in the encoding stage often seems to be the dominant source (French, 1984a, 1984b). The fact that the time constants of adaptation depend strongly and systematically on the firing rate for locust auditory receptors also indicates that spike adaptation is an important mechanism (Benda, 2002). Because spike adaptation takes place after spectral integration, it is independent of the sound frequency. Our model description is not affected by such a frequency-independent adaptation as long as we focus on a fixed response episode. The number of spikes occurring during such an episode is still a function of the effective sound intensity, although the distribution of the spikes may display a certain structure within the response. For different response episodes, the decrease in the firing rate over time is simply reflected in an increase of the filter constants $C_{1}$ and $C_{2}$ by a common factor, which could also be absorbed in the function $r(J)$. In particular, 

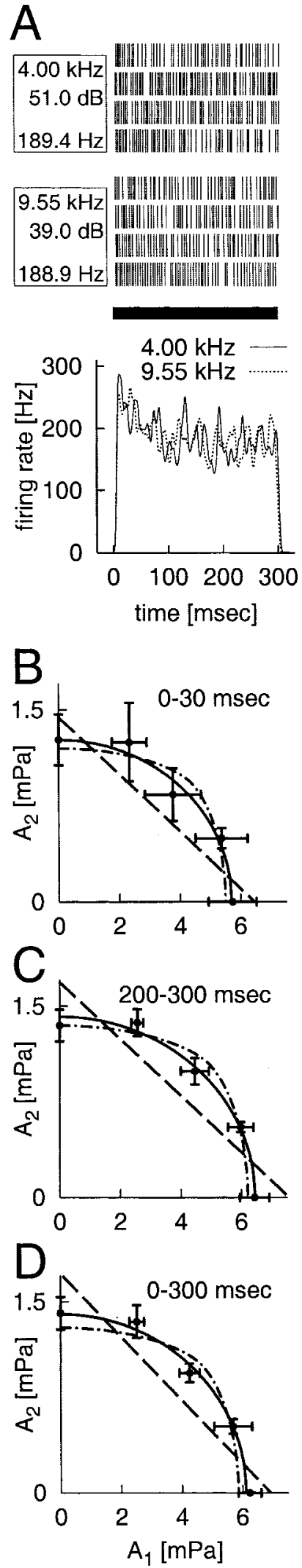

Figure 6. Responses and iso-firing-rate curves for two cells with stimuli of $300 \mathrm{msec}$. Each of the two columns ( $A-D$ and $E-H$, respectively) depicts results from a single cell. $A$ and $E$ show four typical spike trains in response to pure tones with frequencies $f_{1}=4.00 \mathrm{kHz}$ (top) and $f_{2}=9.55$ $\mathrm{kHz}$ (middle) as well as the corresponding instantaneous firing rates the ratios $R=C_{1} / C_{2}$ of the filter constants for the onset, $R_{\mathrm{O}}$, and the steady state, $R_{\mathrm{S}}$, should be the same.

The development of the firing rates in Figure 6 shows that the transient parts of the response are generally similar and that they have approximately the same time constant independent of sound frequency. For the cell that is depicted in the right column of Figure 6, though, the firing rates for the two sound frequencies clearly differ in the first $30 \mathrm{msec}$. This indicates that, on short time scales, the adaptation dynamics can depend on sound frequency, which implies an adaptation mechanism within the coupling or transduction processes. For our model description, such a phenomenon results in a difference between $R_{\mathrm{O}}$ and $R_{\mathrm{S}}$. This can be observed, e.g., in the right column of Figure 6, where the ellipses for the onset (Fig. 6F) and steady state (Fig. 6G) have different shapes.

We analyzed this effect quantitatively for the 10 investigated cells by determining the relative change $\Delta R=\left|R_{\mathrm{O}}-R_{\mathrm{S}}\right| / R_{\mathrm{S}}$. We found values of $\Delta R$ between 1 and $25 \%$, which must be compared with the error measures for the values of $R$ of $\sim 10 \%$. Half of the cells had a $\Delta R$ value that was larger than their noise level. The cell depicted in the right column of Figure 6 showed the largest $\Delta R$ of the 10 cells. The total values of the filter constants $C_{1}$ and $C_{2}$, on the other hand, change between onset and steady state by $10-$ $50 \%$, with error measures of $5-10 \%$. We conclude that all cells that we analyzed were affected by adaptation and that in some cells, a small fraction of the adaptation phenomenon might be attributed to frequency-dependent mechanisms. These frequencydependent effects are restricted to approximately the first 30 msec. Analyzing the time window from 40 to $70 \mathrm{msec}$ after stimulus onset, e.g., gives very similar ratios of $C_{1}$ and $C_{2}$ as for the steady state. Consequently, the frequency-dependent changes are negligible for the model description of the average response to longer stimuli. For example, the area between the two firing rate curves in the first $30 \mathrm{msec}$ of Figure $6 E$ (bottom), which denotes the difference in spike count attributable to the frequency dependence, corresponds to only $\sim 2 \%$ of the total spike count. For the remaining part of this study, we therefore use the full responses to $100 \mathrm{msec}$ stimuli, for which it is easier to collect a sufficient amount of data in the limited recording time.

(bottom). The sound frequency in kilohertz and the intensity in decibels of the stimulus as well as the elicited firing rate in hertz are indicated in the boxes to the left of the spike trains. The sound intensities for which the responses are shown were chosen such that the average firing rates approximately coincided for the two sound frequencies. The duration of the stimuli is denoted by the thick bars. The instantaneous firing rates were calculated by averaging over the inverse interspike intervals at each point in time and subsequently smoothed with a Gaussian of $2 \mathrm{msec}$ SD. One observes a strong transient in the first $30-100 \mathrm{msec}$. In addition, the cell depicted in $E-H$ exhibited a slightly reduced firing rate in the first few milliseconds for the $4 \mathrm{kHz}$ tone compared with the $9.55 \mathrm{kHz}$ tone. $B-D$ and $\mathrm{F}-\mathrm{H}$ show iso-firing-rate data and fits of the three hypotheses for the two cells obtained from different episodes of the responses. The time window used for the analysis is denoted in each of the panels. $B$ and $F$ capture the onset response of the first $30 \mathrm{msec}$. $C$ and $G$ refer to the steady state, and $D$ and $H$ refer to the total response. The ellipses corresponding to the energy hypothesis (solid lines) lead to notedly better fits of the data than the curves for the amplitude hypothesis (dashed lines) and the pressure hypothesis (dash-dotted lines), regardless of the analyzed response window. For the cell illustrated in the right column, the ellipse for the onset response $(F)$ has a half-axes ratio, $R_{\mathrm{O}}=0.63$, that differs by $\sim 25 \%$ from that for the steady state $(G), R_{\mathrm{S}}=0.84$, and by $\sim 15 \%$ from that for the total response $(H), R_{\text {total }}=0.74$. On the other hand, the half-axes ratios for the cell in the left column vary by $<5 \%\left(R_{\mathrm{O}}=4.51\right.$, $R_{S}=4.55$, and $R_{\text {total }}=4.36$ ). 


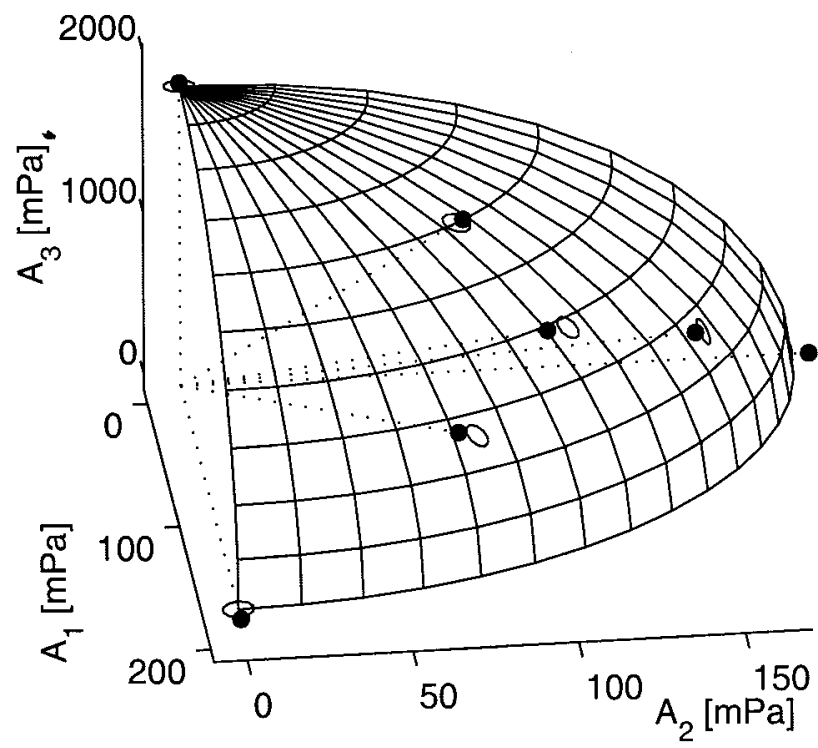

Figure 7. Iso-firing-rate surface for superpositions of three pure tones for one receptor cell. Amplitude triplets resulting in a firing rate of 150 $\mathrm{Hz}$ are shown as filled circles. The three-dimensional mesh displays an ellipsoid with the three half-axes fitted to the data and illustrates the prediction for the iso-firing-rate surface from the energy hypothesis. The filter constants obtained from the fit are $C_{1}=0.172 \mathrm{~Pa}, C_{2}=0.186 \mathrm{~Pa}$, and $C_{3}=1.88 \mathrm{~Pa}$. For optical guidance, the measured points are connected to the origin of the coordinate system by dotted lines. The intersection points of these lines with the ellipsoid are portrayed by open circles on the ellipsoid. For clarity, the iso-firing-rate surfaces corresponding to the amplitude and pressure hypotheses are not shown.

\section{Superpositions of three pure tones}

To see whether the findings from the two-tone experiments generalize to sounds with more complex frequency spectra, responses to superpositions of three pure tones were analyzed. We applied the same approach as for the two-tone experiments with $100 \mathrm{msec}$ stimuli and identified iso-firing-rate surfaces in the three-dimensional subspace $\left(A_{1}, A_{2}, A_{3}\right)$. The three hypotheses yield predictions about these surfaces in the form of a plane (amplitude hypothesis), an ellipsoid (energy hypothesis), and a more strongly bent surface (pressure hypothesis) the exact shape of which has to be determined numerically.

Responses to superpositions of three pure tones were measured for eight cells. From the rate-intensity functions, we determined amplitude triplets corresponding to a firing rate of $150 \mathrm{~Hz}$. Figure 7 illustrates the results for one cell and also shows the fitted ellipsoid corresponding to the iso-firing-rate surface of the energy hypothesis. We applied a $\chi^{2}$ test and found that the amplitude hypothesis is rejected at the $1 \%$ level for all eight cells, whereas the energy hypothesis is rejected for one cell and the pressure hypothesis is rejected for four cells. We again compared the fits for the energy and the pressure hypothesis in more detail. In all cases, the energy hypothesis gave a lower $\chi^{2}$ than the pressure hypothesis, and the Bayesian estimate of the probability of the model given the data again strongly favored the energy hypothesis over the pressure hypothesis [mean of $p(\mathrm{EH} \mid$ data $)$ was 0.916 with $0.109 \mathrm{SD}$, median $0.987, N=8$ ]. Thus, spectral integration for three pure tones is also best described by the energy hypothesis, whereas the amplitude and the pressure hypothesis are rejected by the data.

\section{Comparison of pure-tone and noise stimuli}

So far we have found that the energy hypothesis describes spectral integration of mixtures of two and three pure tones. We now pose the question whether this hypothesis also applies to stimuli composed of many frequencies. In particular, we aim at predicting the response to a bandpass-filtered Gaussian white noise based on the knowledge of the filter constants $C_{\mathrm{n}}$ and a pure-tone rateintensity function. Spike-train responses to the noise stimulus have the same structure as responses to pure-tone stimulation (data not shown). We therefore again focus on the firing rate and measure rate-intensity functions for the noise stimulus. Our model predicts that these should have the same shape as the pure-tone rate-intensity functions (see Materials and Methods). The expected distance $\Delta I$ between the two rate-intensity functions can be calculated if the filter constants and the power spectrum of the noise stimulus are known. The values for the energy hypothesis, $\Delta I_{\mathrm{EH}}$, and the pressure hypothesis, $\Delta I_{\mathrm{PH}}$, are given in Equations 10 and 11.

The pure-tone stimulus has a frequency of $4 \mathrm{kHz}$, and the noise stimulus is bandpass filtered between 5 and $10 \mathrm{kHz}$, a region in which many receptors are most sensitive. Rate-intensity functions for these two types of stimuli were measured for 10 cells. In addition, filter constants in the range of $5-10 \mathrm{kHz}$ were determined independently by measuring the amplitudes of pure tones leading to a firing rate of $260 \mathrm{~Hz}$. Figure 8 shows rate-intensity functions for the pure-tone as well as the noise stimulus together with the predictions that are obtained from shifting the pure-tone rate-intensity functions by $\Delta I_{\mathrm{EH}}$. In each case, the two measured rate-intensity functions are almost identical in shape, as expected from the model. Furthermore, the measured noise-stimulus rateintensity function and the shifted pure-tone rate-intensity function coincide closely in most cases. Note that only results from pure-tone stimulation are used for the prediction of the noisesignal responses. To assess the results quantitatively, we calculated the deviation of $\Delta I_{\mathrm{EH}}$ from the actual distance between the rate-intensity functions, $\Delta I_{\text {true }}$, in each case. For the energy hypothesis, $\Delta I_{\mathrm{EH}}-\Delta I_{\text {true }}$ has a mean of $-0.62 \pm 0.68 \mathrm{~dB}(\mathrm{SE})$. The spread of these data $(\mathrm{SD}$ of $2.16 \mathrm{~dB})$ corresponds to the expected measurement accuracy, which can be estimated to be $\sim 2 \mathrm{~dB}$; the determination of $C^{\mathrm{pt}}$, the collection of $C_{\mathrm{n}}$, and the locations of both $r^{\mathrm{pt}}(I)$ and $r^{\text {noise }}(I)$ all contribute independently with $\sim 1 \mathrm{~dB}$ error range. The pressure hypothesis yields $\Delta I_{\mathrm{PH}}-$ $\Delta I_{\text {true }}$ with a mean of $0.43 \pm 0.68 \mathrm{~dB}(\mathrm{SE})$ and is thus not ruled out by this experiment.

The results suggest that the description of spectral integration by the energy hypothesis, as derived from the two- and three-tone experiments, is also applicable to more complex stimuli. The model can be used for an accurate prediction of the location of the rate-intensity function after measuring the filter constants from pure-tone responses. The predictability of actual firing rates, however, is limited by the steepness of the rate-intensity functions. Because the range between threshold and saturation usually spans only $\sim 15-30 \mathrm{~dB}$, small inaccuracies of a few decibels about the prediction of the shift between the rate-intensity functions have a strong effect on individual firing-rate predictions.

\section{DISCUSSION}

Spectral integration is an important feature of auditory encoding and is closely connected to the mechanosensory transduction process. Our data show that the response of locust auditory receptor cells to stationary sound stimuli is determined by an "effective sound intensity" $J$ that can be calculated from the 


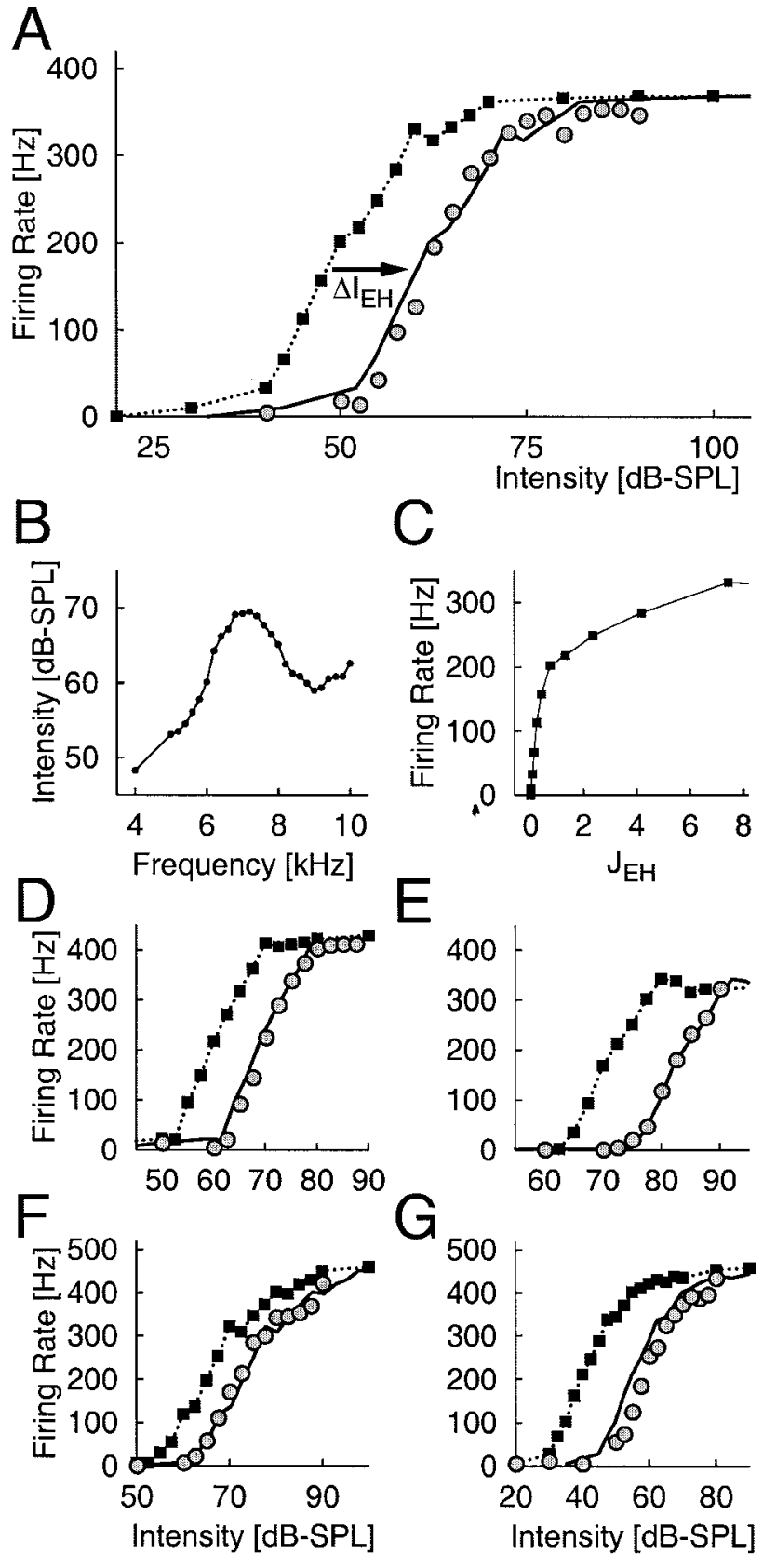

Figure 8. Comparison of the predictions for a noise-signal rate-intensity function with the actual measurement. $A$, Predicted and measured rateintensity functions. The squares connected by the dotted line depict a rate-intensity function for a $4 \mathrm{kHz}$ tone measured for one receptor cell. Using the energy hypothesis and the measured filter constants $C_{n}$, a prediction for the rate-intensity function of a noise signal (bandpass filtered between 5 and $10 \mathrm{kHz}$ ) is derived (solid line). It is obtained by shifting the pure-tone rate-intensity function by an intensity $\Delta I_{\mathrm{EH}}=12.1$ $\mathrm{dB}$ as indicated by the arrow. The measured firing rate of the receptor cell in response to the noise signal is shown by the circles. Data and model prediction agree well in both the overall shape of the rate-intensity function and the location on the intensity axis. The true shift between the measured rate-intensity function is estimated as $\Delta I_{\text {true }}=12.6 \mathrm{~dB}$. $B$, Determination of filter constants. The filled circles depict the measured intensities for pure tones between 4 and $10 \mathrm{kHz}$ that led to a firing rate of $260 \mathrm{~Hz}$ in each case. These data were used to determine the filter constants $C_{n}$ for the $4 \mathrm{kHz}$ pure tone as well as for the range from 5 to 10 $\mathrm{kHz}$. Further filter constants in this range were obtained by linear interpolation of this curve. $C$, The response function $r\left(J_{\mathrm{EH}}\right)$ as determined by the rate-intensity function for the $4 \mathrm{kHz}$ tone. The same firing rates that result in the squares in $A$ are plotted against the effective sound intensity stimulus spectrum and the sensitivity of the receptor at different sound frequencies. The sound-intensity coding of the receptor cells can thus be described in a three-step process. First, the tympanal membrane acts as a linear filter. The relevant characteristics of the filter can be determined using pure-tone stimuli by measuring which intensities correspond to a given firing rate of the receptor. Second, the effective sound intensity $J$ is obtained by summing up all energies contained in the individual frequency components of the filtered signal. We believe that this summation reflects the dynamic properties of the mechanosensory transduction channels. Ultimately, a biophysical investigation is required to confirm this view. In a final step, the effective sound intensity is put through a nonlinear response function independent of the spectral contents of the original signal. The shape of this response function can be derived from the measurement of a single rateintensity function with arbitrary but fixed spectral content.

Alternative hypotheses that compute $J$ as the maximum amplitude or the integrated pressure are rejected by the analysis of the responses of the receptors to superpositions of two or three pure tones. Although the amplitude hypothesis can be clearly discarded, the energy and pressure hypotheses are more similar in their predictions regarding spectral integration. However, the combined evidence from several statistical investigations demonstrates that the pressure hypothesis fails in several single cases and that the energy hypothesis provides a far better description of the data.

Comparison between responses to pure tones and bandpassfiltered noise shows that the energy hypothesis also accounts for the responses to more complex stimuli. The model can therefore be used to accurately predict the rate-intensity functions for noise-like signals.

\section{Effects of stimulus onset and adaptation}

A more detailed analysis reveals that the energy hypothesis also describes spectral integration for the onset and the steady-state responses individually. The model parameters depend on the response episode investigated. The main effect is a change in the filter constants by a common factor attributable to spikedependent adaptation. For a generalization of the model to fluctuating stimuli, this dependence of the parameters on the adaptation state could be explicitly incorporated, e.g., by using the generic model of (Benda et al., 2001; Benda, 2002). In some cells, changes in the ratio of the filter constants between onset and steady state suggest additional, although smaller sound-frequencydependent dynamics of the adaptation. Besides the dominant spike adaptation, there might thus be a second adaptation phenomenon, which occurs before spectral integration. It might be caused by either a mechanical effect of the vibrations of the tympanum and

$\leftarrow$

$J_{\mathrm{EH}}$ of the energy hypothesis. $J_{\mathrm{EH}}$ is given by $1 / 2 \cdot A^{2} / C^{2}$, where $A$ denotes the amplitude of the pure tone and $C$ denotes the filter constant, which is determined by the intensity of the pure tone that drives the cell at $260 \mathrm{~Hz}$. Although the pure-tone rate-intensity function displayed in $A$ has a large nearly linear section from $\sim 40$ to $60 \mathrm{~dB}$ SPL, the response function $r\left(J_{\mathrm{EH}}\right)$ is clearly nonlinear in the corresponding region (from $J_{\mathrm{EH}}=0.08$ to $J_{\mathrm{EH}}=$ 8 ) and resembles a square-root function. $D-G$, Predicted and measured rate-intensity functions for the noise signal from four other cells. Symbols are used as in $A$. Note the different scales on the axes. Accordingly, the slopes of the rate-intensity functions differ considerably from cell to cell, but for a single cell, they are almost identical for pure-tone and noise stimulation. The values for $\Delta I_{\mathrm{EH}}$ and $\Delta I_{\text {true }}$ in these four cases are $D$, $\Delta I_{\mathrm{EH}}=9.0 \mathrm{~dB}, \Delta I_{\text {true }}=9.8 \mathrm{~dB}, E, \Delta I_{\mathrm{EH}}=12.1 \mathrm{~dB}, \Delta I_{\text {true }}=11.8 \mathrm{~dB}, F$, $\Delta I_{\mathrm{EH}}=7.8 \mathrm{~dB}, \Delta I_{\text {true }}=6.6 \mathrm{~dB}, G, \Delta I_{\mathrm{EH}}=14.9 \mathrm{~dB}, \Delta I_{\text {true }}=18.4 \mathrm{~dB}$. 
associated structures or a property of the transduction channels. Because the effect is generally small and restricted to the first 30 msec, it can be neglected for the description of the average response to longer stimuli, which was the focus of the present study.

\section{Conceptual framework}

Combining the two concepts "spectral integration" and "isofiring-rate regions" allowed us to rigorously compare different transduction models. A key ingredient in the experimental procedure was the systematic exploration of regions of constant activity under variation of the stimulus composition, in the present case the spectrum of a sound signal. Investigating such regions implies a change of the traditional perspective regarding neural input-output relations. Instead of asking what output is produced by a given input, one seeks to identify input ensembles that are associated with a fixed output. Reliable on-line analysis and automatic feedback to the stimulus generation are a central aspect of this approach. Based on increasingly available highspeed computing power, the method could be easily extended to identify more general invariant regions in auditory and other stimulus domains.

Our framework may be compared with the technique of silent substitution (Estévez and Spekreijse, 1982), in which the spectral composition of a visual stimulus is varied systematically such that the resulting stimuli always lead to the same activity of one (or more) receptor types in the retina. Fluctuations in visually evoked potentials can then be interpreted as being caused by the remaining receptors. In this case, however, the iso-activity regions of the receptors are not explored, but must be known accurately beforehand, and they are not compared with alternative model predictions.

\section{Comparison with studies of temporal integration in other auditory systems}

Our results go along well with the fact that temporal energy integration describes firing thresholds for double-click and intensity-duration trade-off experiments in receptor cells of moths (Tougaard, 1996, 1998). If this finding can also be confirmed for locust auditory receptors, spectral and temporal integration could be combined in a single simple model. Trade offs between intensity and duration would then be expected to occur for stimuli on time scales of a few milliseconds, the apparent integration time of the receptors, which is well below the stimulus durations used in this study. In mammalian auditory nerve fibers, on the other hand, first-spike latencies correspond to the integrated pressure and not the energy (Heil and Neubauer, 2001). It is possible that this is caused by a fundamental difference in the transduction mechanisms of hair cells and insect auditory receptors. However, latency measurements reflect properties of the transduction as well as properties of additional dynamic processes, such as synaptic transmission, internal calcium dynamics, and spike generation. In this context, it should be noted that the latency in type I excitable membranes depends strongly and nonlinearly on the input strength (Hodgkin, 1948; Rinzel and Ermentrout, 1998; Izhikevich, 2000). This opens up the possibility that properties of the spike generator alter the effective input in such a way that energy integration is in accordance with the observed correspondence between latency and the temporal pressure integral. In fact, Ermentrout (1996) showed that in type I membranes, the firing rate $r$ to a constant stimulus $S$ above the firing threshold $S^{*}$ approximately obeys the square-root relation $r(S) \sim \sqrt{S-S^{*}}$. For a simplified phase-integrator model (Hoppensteadt, 1997), the latency $\Delta t$ is then given by the condition that the integral $\int_{0}^{\Delta t} \sqrt{S(t)-S^{*}} d t$ reaches a threshold value. According to the energy hypothesis, $S$ is proportional to the square of the pressure amplitude $A$ of a pure tone and in most cases large compared with $S^{*}$. This cancels the square root, thus resulting in the latency condition $\int_{0}^{\Delta t} A(t) d t=$ const, the dominant component of the model proposed by Heil and Neubauer (2001). The above considerations may also explain the apparent discrepancy between the latency measurements and the fact that psycho-acoustic studies successfully apply energy-integration models (Garner, 1947; Plomp and Bouman, 1959; Zwislocki, 1965; Florentine et al., 1988). Further experiments are needed to decide this, however.

Response properties of hair cells and mammalian auditory nerve fibers are complicated by mechanical nonlinearities induced by the cochlea and a more intricate signal pathway than is the case in insect auditory systems. Nevertheless, measurements of basilar-membrane vibrations indicate that outside a region around the characteristic frequency, the stimulus coupling to mammalian auditory receptors occurs in an approximately linear fashion (Eguíluz et al., 2000; Ruggero et al., 2000). This suggests that a phenomenological study along the lines of the present investigation might also reveal interesting properties of the transduction process in hair cells.

\section{Implications for the locust auditory system}

Practical implications of our results include a more reliable characterization of insect auditory receptor sensitivity by measuring the intensities necessary to provoke a given non-zero firing rate instead of the threshold curve. The latter is notoriously difficult to measure because the rate-intensity functions usually flatten out near the threshold and are corrupted by background activity (Michelsen, 1971c). At least in locust receptor cells, the threshold curve runs approximately parallel to any other curve of equal response, and a single additional rate-intensity function can determine the distance between the measured curve and the actual threshold curve. Furthermore, our results show that average responses of an auditory receptor to complex stimuli can be well predicted once the cell-specific effective sound intensity $J$ has been measured. The resulting quantitative correspondence between the stimulus spectrum and the firing rate differs from the predictions of an earlier heuristic approach (Lang, 2000). Our result will thus be helpful for systematic investigations of the processing of natural communication signals, such as grasshopper calling songs (Machens et al., 2001).

\section{Linear versus nonlinear models}

The simplicity of our model, which is linear up to a final static nonlinearity, is consistent with the fact that previous studies have found no indications of dominant nonlinearities or active movement of the sensory cilia (cf. Eberl, 1999). Distortion-product otoacoustic emissions from locust ears indicate slight nonlinearities at the tympanal membrane, but only at $\sim 50 \mathrm{~dB}$ below the stimulating intensities (Kössl and Boyan, 1998). Many other auditory systems, on the other hand, are strongly affected by nonlinear mechanisms and active signal amplification leading to increased sensitivity and frequency resolution. This phenomenon is common in vertebrate ears (Fettiplace and Fuchs, 1999; Hudspeth et al., 2000) but has also been shown to exist in some insect auditory systems (Göpfert and Robert, 2001).

\section{Implications for other mechanosensory systems}

It can be speculated that the nonlinearities mentioned above are additional features on top of the same underlying mechanosensory transduction process. Recent findings of structural and func- 
tional similarities between hair cells and the Drosophila sensory bristle as well as the discovery in Drosophila of homologs of mammalian genes related to hearing and deafness support this view and suggest that many aspects of mechanosensory transduction among insects and vertebrates are conserved (Adam et al., 1998; Bermingham et al., 1999; Eberl, 1999; Fritzsch et al., 2000; Walker et al., 2000; Gillespie and Walker, 2001). The energy hypothesis might thus be extended to account for spectral integration in other mechanosensory systems as well, possibly after modifications that take the system-specific nonlinearities explicitly into account.

Mechanosensory transduction is also involved in a wide range of other senses, including touch, proprioception, and the sense of balance. Unlike transduction mechanisms that involve secondmessenger signaling suited for biochemical analysis, mechanosensory changes of the membrane conductance result from a direct coupling with the mechanical stimulus: stretch, compression of the cell, or deflection of associated processes or cilia (Corey and Hudspeth, 1979; Hudspeth, 1985; Hudspeth and Logothetis, 2000). This direct and fast electrophysiological response has so far resisted a detailed biophysical analysis (Gillespie, 1995). Our method of finding regions of constant neural response for varying spectral composition provides a novel approach for distinguishing between different hypotheses about receptor integration, sets quantitative constraints that any future biophysical model has to satisfy, and is applicable to a wide range of other (mechano)sensory systems.

\section{APPENDIX: CALCULATION OF THE INTENSITY SHIFT BETWEEN PURE-TONE AND NOISE SIGNALS}

We denote the effective sound intensities of the noise signal by $J_{\mathrm{EH}}^{\text {noise }}$ and $J_{\mathrm{PH}}^{\text {noise }}$ and the effective sound intensities of the puretone signal by $J_{\mathrm{EH}}^{\mathrm{pt}}$ and $J_{\mathrm{PH}}^{\mathrm{pt}}$ according to the energy and pressure hypotheses, respectively. The intensity in the decibel SPL scale is defined as:

$$
I=20 \log _{10} \frac{\sqrt{\left\langle S(t)^{2}\right\rangle}}{A_{0}}
$$

with $A_{0}=20 \mu \mathrm{Pa}$. For the noise signal:

$$
S^{\text {noise }}(t)=\sum_{n} A_{n} \sin \left(2 \pi f_{n} t+\varphi_{n}\right),
$$

the root-mean-square is obtained as:

$$
\sqrt{\left\langle S(t)^{2}\right\rangle}=\sqrt{\frac{1}{2} \sum_{n} A_{n}^{2}}
$$

which implies that the intensity is given by:

$$
I^{\text {noise }}=20 \log _{10} \frac{\sqrt{\frac{1}{2} \sum_{n} A_{n}^{2}}}{A_{0}} .
$$

The effective sound intensity of the energy hypothesis, Equation 17 , can thus be written as:

$$
J_{\mathrm{EH}}^{\mathrm{noise}}=\frac{1}{2} \sum_{n} \frac{A_{n}^{2}}{C_{n}^{2}}=10^{\text {Inoise } / 10} \cdot A_{0}^{2} \cdot \frac{\sum_{n} A_{n}^{2} / C_{n}^{2}}{\sum_{n} A_{n}^{2}},
$$

where the dependence on the intensity is given explicitly because the term

$$
\sum \frac{A_{n}^{2}}{C_{n}^{2}} / \sum A_{n}^{2}
$$

is invariant to intensity changes.

For $J_{\mathrm{PH}}^{\text {noise }}$, we note that the values of $\tilde{S}(t)$ are distributed according to a Gaussian distribution with variance $\sigma^{2}$ that is given by:

$$
\sigma^{2}=\frac{1}{2} \sum_{n} \frac{A_{n}^{2}}{C_{n}^{2}}=J_{\mathrm{EH}}^{\mathrm{noise}} .
$$

For a Gaussian distribution with $\mathrm{SD} \sigma$, the mean of the absolute value can be calculated as

$$
\langle|\tilde{S}(t)|\rangle=\sqrt{\frac{2}{\pi}} \cdot \sigma,
$$

and we therefore obtain from Equation 18:

$$
J_{\mathrm{PH}}^{\text {noise }}=\sqrt{\frac{2}{\pi} \cdot J_{\mathrm{EH}}^{\text {noise }}}=\sqrt{\frac{2}{\pi}} \cdot 10^{I^{\text {noise }} / 20} \cdot A_{0} \cdot \sqrt{\frac{\sum_{n} A_{n}^{2} / C_{n}^{2}}{\sum_{n} A_{n}^{2}}} .
$$

Equivalently, we find for the pure-tone stimulus $S^{\mathrm{pt}}(\mathrm{t})=A^{\mathrm{pt}}$ $\sin (2 \pi f t)$ :

$$
\begin{gathered}
J_{\mathrm{EH}}^{\mathrm{pt}}=\frac{1}{2} \cdot \frac{A^{\mathrm{pt}^{2}}}{C^{\mathrm{pt}^{2}}}=10^{I \mathrm{pt} / 10} \cdot \frac{A_{0}^{2}}{C^{\mathrm{pt} 2}}, \\
J_{\mathrm{PH}}^{\mathrm{pt}}=\frac{2}{\pi} \cdot \frac{A^{\mathrm{pt}}}{C^{\mathrm{pt}}}=\frac{2 \sqrt{2}}{\pi} \cdot 10^{I \mathrm{pt} / 20} \cdot \frac{A_{0}}{C^{\mathrm{pt}}},
\end{gathered}
$$

where $C^{\mathrm{pt}}$ denotes the filter constant for the pure tone. These latter relationships can be inverted to yield $I^{\mathrm{pt}}$ as a function of $J^{\mathrm{pt}}$. Because equal $J$ implies equal firing rate, we can then substitute $J^{\mathrm{pt}}$ by $J^{\text {noise }}$ to obtain that intensity of the pure tone that leads to the same firing rate as a given intensity of the noise signal:

$$
\begin{aligned}
r_{\mathrm{EH}}^{\mathrm{noise}}\left(I^{\text {noise }}\right) & =r_{\mathrm{EH}}^{\mathrm{pt}}\left(10 \log _{10}\left(\frac{\left(C^{\mathrm{pt}}\right)^{2}}{A_{0}^{2}} \cdot J_{\mathrm{EH}}^{\text {noise }}\right)\right) \\
& =r_{\mathrm{EH}}^{\mathrm{pt}}\left(I^{\mathrm{noise}}+10 \log _{10}\left(\left(C^{\mathrm{pt}}\right)^{2} \frac{\sum_{n} A_{n}^{2} / C_{n}^{2}}{\sum_{n} A_{n}^{2}}\right)\right), \\
r_{\mathrm{PH}}^{\mathrm{noise}}\left(I^{\text {noise }}\right) & =r_{\mathrm{PH}}^{\mathrm{pt}}\left(20 \log _{10}\left(\frac{\pi}{2 \sqrt{2}} \cdot \frac{C^{\mathrm{pt}}}{A_{0}} \cdot J_{\mathrm{PH}}^{\mathrm{noise}}\right)\right) \\
& =r_{\mathrm{PH}}^{\mathrm{pt}}\left(I^{\text {noise }}+10 \log _{10}\left(\frac{\pi}{4}\left(C^{\mathrm{pt}}\right)^{2} \frac{\sum_{n} A_{n}^{2} / C_{n}^{2}}{\sum_{n} A_{n}^{2}}\right)\right) .
\end{aligned}
$$

From these formulas, we can directly read out $\Delta I$ for the two hypotheses by comparison with Equation 8.

\section{REFERENCES}

Adam J, Myat A, Le Roux I, Eddison M, Henrique D, Ish-Horowicz D, Lewis J (1998) Cell fate choices and the expression of Notch, Delta and Serrate homologues in the chick inner ear: parallels with Drosophila sense-organ development. Development 125:4645-4654.

Barlow R (1989) Statistics. New York: Wiley.

Benda J (2002) Single neuron dynamics-models linking theory and experiment. PhD thesis, Humboldt University Berlin.

Benda J, Bethge M, Hennig M, Pawelzik K, Herz AVM (2001) Spikefrequency adaptation: phenomenological model and experimental tests. Neurocomputing 38-40:105-110.

Bermingham NA, Hassan BA, Price SD, Vollrath MA, Ben-Arie N, Eatock RA, Bellen HJ, Lysakowski A (1999) Math1: an essential gene for the generation of inner hair cells. Science 284:1837-1841. 
Chapman KM, Mosinger JL, Duckrow RB (1979) The role of distributed viscoelastic coupling in sensory adaptation in an insect mechanoreceptor. J Comp Physiol 131:1-12.

Corey DP, Hudspeth AJ (1979) Ionic basis of the receptor potential in a vertebrate hair cell. Nature 281:675-677.

Eberl DF (1999) Feeling the vibes: chordotonal mechanisms in insect hearing. Curr Opin Neurobiol 9:389-393.

Eguíluz VM, Ospeck M, Choe Y, Hudspeth AJ, Magnasco MO (2000) Essential nonlinearities in hearing. Phys Rev Lett 84:5232-5235.

Ermentrout B (1996) Type I membranes, phase resetting curves, and synchrony. Neural Comput 8:979-1001.

Estévez O, Spekreijse H (1982) The "silent substition" method in visual research. Vision Res 22:681-691.

Eyzaguirre C, Kuffler SW (1955) Processes of excitation in the dendrites and in the soma of single isolated sensory nerve cells of the lobster and crayfish. J Gen Physiol 39:87-119.

Fettiplace R, Fuchs PA (1999) Mechanics of hair cell tuning. Annu Rev Physiol 61:809-834.

Florentine M, Fastl H, Buus S (1988) Temporal integration in normal hearing, cochlear impairment, and impairment simulated by masking. J Acoust Soc Am 84:195-203.

French AS (1984a) Action potential adaptation in the cockroach tactile spine. J Comp Physiol [A] 155:803-812.

French AS (1984b) The receptor potential and adaptation in the cockroach tactile spine. J Neurosci 4:2063-2068.

French AS (1992) Mechanotransduction. Annu Rev Physiol 54:135-152.

Fritzsch B, Beisel KW, Bermingham NA (2000) Developmental evolutionary biology of the vertebrate ear: conserving mechanoelectric transduction and developmental pathways in diverging morphologies. NeuroReport 11:R35-R44

Garner WR (1947) The effect of frequency spectrum on temporal integration of energy in the ear. J Acoust Soc Am 19:808-814.

Gillespie PG (1995) Molecular machinery of auditory and vestibular transduction. Curr Opin Neurobiol 5:449-455.

Gillespie PG, Walker RG (2001) Molecular basis of mechanosensory transduction. Nature 413:194-202.

Göpfert MC, Robert D (2001) Active auditory mechanics in mosquitoes. Proc R Soc Lond B Biol Sci 268:333-339.

Gray EG (1960) The fine structure of the insect ear. Philos Trans R Soc Lon B Biol Sci 243:75-94.

Heil P, Neubauer H (2001) Temporal integration of sound pressure determines thresholds of auditory-nerve fibers. J Neurosci 21:7404-7415.

Hill KG (1983a) The physiology of locust auditory receptors. I. Discrete depolarizations of receptor cells. J Comp Physiol [A] 152:475-482.

Hill KG (1983b) The physiology of locust auditory receptors. II. Membrane potentials associated with the response of the receptor cell. J Comp Physiol [A] 152:483-493.

Hodgkin A (1948) The local electric changes associated with repetitive action in a non-medullated axon. J Physiol (Lond) 107:165-181.

Holt JR, Corey DP (2000) Two mechanisms for transducer adaptation in vertebrate hair cells. Proc Natl Acad Sci USA 97:11730-11735.

Hoppensteadt FC (1997) An Introduction to the Mathematics of Neurons, modeling in the Frequency Domain, Ed 2. Cambridge, UK: Cambridge UP

Hudspeth AJ (1985) The cellular basis of hearing: the biophysics of hair cells. Science 230:745-752.

Hudspeth AJ, Logothetis NK (2000) Sensory systems. Curr Opin Neurobiol 10:631-641.

Hudspeth AJ, Choe Y, Mehta AD, Martin P (2000) Putting ion channels to work: mechanoelectric transduction, adaptation, and amplification by hair cells. Proc Natl Acad Sci USA 97:11765-11772.

Izhikevich EM (2000) Neural excitability, spiking, and bursting. Int J Bifurcat Chaos 10:1171-1266.
Jacobs K, Otte B, Lakes-Harlan R (1999) Tympanal receptor cells of Schistocerca gregaria: correlation of soma positions and dendrite attachment sites, central projections and physiologies. J Exp Zool 283:270-285.

Koch C (1999) Biophysics of Computation. New York: Oxford UP.

Kössl M, Boyan GS (1998) Acoustic distortion products from the ear of a grasshopper. J Acoust Soc Am 104:326-335.

Lang F (2000) Acoustic communication distances of a gomphocerine grasshopper. Bioacoustics 10:233-258.

Machens CK, Stemmler MB, Prinz P, Krahe R, Ronacher B, Herz AVM (2001) Representation of acoustic communication signals by insect auditory receptor neurons. J Neurosci 21:3215-3227.

Matthews PBC, Stein RB (1969) The sensitivity of muscle spindle afferents to sinusoidal stretching. J Physiol (Lond) 200:723-743.

Michelsen A (1971a) The physiology of the locust ear. I. Frequency sensitivity of single cells in the isolated ear. Z Vgl Physiol 71:49-62.

Michelsen A (1971b) The physiology of the locust ear. II. Frequency discrimination based upon resonance in the tympanum. Z Vgl Physiol 71:63-101.

Michelsen A (1971c) The physiology of the locust ear. III. Acoustic properties of the intact ear. Z Vgl Physiol 71:102-128.

Michelsen A (1979) Insect ears as mechanical systems. Am Sci 67:696-706.

Michelsen A, Rohrseitz K (1995) Directional sound processing and interaural sound transmission in a small and a large grasshopper. J Exp Biol 198:1817-1827.

Plomp R, Bouman MA (1959) Relation between hearing threshold and duration for tone pulses. J Acoust Soc Am 31:749-758.

Press WH, Teukolsky SA, Vetterling WT, Flannery BP (1992) Numerical recipes. Cambridge, UK: Cambridge UP.

Purali N, Rydqvist B (1998) Action potential and sodium current in the slowly and rapidly adapting stretch receptor neurons of the crayfish (Astacus astacus). J Neurophysiol 80:2121-2132.

Ricci AJ, Wu Y-C, Fettiplace R (1998) The endogenous calcium buffer and the time course of transducer adaptation in auditory hair cells. J Neurosci 18:8261-8277.

Rinzel J, Ermentrout B (1998) Analysis of neural excitability and oscillations. In: Methods in neural modeling: from ions to networks, Ed 2 (Koch C, Segev I, eds), pp 251-292. Cambridge, MA: MIT.

Römer H (1976) Die Informationsverarbeitung tympanaler Rezeptorelemente von Locusta migratoria (acrididae, orthoptera). J Comp Physiol 109:101-122.

Römer H (1985) Anatomical representation of frequency and intensity in the auditory system of Orthoptera. In: Acoustic and vibrational communication in insects (Kalmring K, Elsner N, eds), pp 25-33. Berlin: Paul Parey.

Ruggero MA, Narayan SS, Temchin AN, Recio A (2000) Mechanical bases of frequency tuning and neural excitation at the base of the cochlea: comparison of basilar-membrane vibrations and auditory-nerve-fiber responses in chinchilla. Proc Natl Acad Sci USA 97:11744-11750.

Schiolten P, Larsen ON, Michelsen A (1981) Mechanical time resolution in some insect ears. J Comp Physiol 143:289-295.

Suga N (1960) Peripheral mechanism of hearing in locust. Jpn J Physiol 10:533-546.

Tougaard J (1996) Energy detection and temporal integration in the noctuid A1 auditory receptor. J Comp Physiol [A] 178:669-677.

Tougaard J (1998) Detection of short pure-tone stimuli in the noctuid ear: what are temporal integration and integration time all about? J Comp Physiol [A] 183:563-572.

Walker RG, Willingham AT, Zuker CS (2000) A Drosophila mechanosensory transduction channel. Science 287:2229-2234.

Zwislocki J (1965) Analysis of some auditory characteristics. In: Handbook of mathematical psychology, Vol 3 (Luce RD, Bush RR, Galanter E, eds), pp 1-97. New York: Wiley. 